\title{
Assessment of Higher-order RANS Closures in a Decelerated Planar Wall-bounded Turbulent Flow
}

\author{
Elbert Jeyapaul* \\ National Institute of Aerospace, Hampton, VA 23666 \\ Gary N. Coleman ${ }^{\dagger}$ and Christopher L. Rumsey ${ }^{\ddagger}$ \\ NASA Langley Research Center, Hampton, VA 23681
}

\begin{abstract}
A reference DNS database is presented, which includes third- and fourth-order moment budgets for unstrained and strained planar channel flow. Existing RANS closure models for third- and fourth-order terms are surveyed, and new model ideas are introduced. The various models are then compared with the DNS data term by term using a priori testing of the higher-order budgets of turbulence transport, velocity-pressure-gradient, and dissipation for both the unstrained and strained databases. Generally, the models for the velocity-pressure-gradient terms are most in need of improvement.
\end{abstract}

\section{Nomenclature}

$\begin{array}{ll}A_{i j} & \text { rate of applied strain } \\ \mathcal{D} & \text { viscous diffusion } \\ D_{t} & \text { material derivative with respect to mean velocities } \\ \partial_{t} & \text { time derivative } \\ \mathcal{T} & \text { turbulent transport } \\ \mathcal{P}^{S} & \text { production due to strain } \\ \mathcal{P}^{T} & \text { production due to Reynolds-stress } \\ \mathcal{P}^{A} & \text { production due to applied strain } \\ \rho & \text { density of fluid } \\ \Pi & \text { velocity-pressure-gradient correlation } \\ \phi & \text { pressure-strain correlation } \\ \psi & \text { pressure-transport } \\ \varepsilon & \text { dissipation } \\ K_{2} & \text { turbulent kinetic energy, } \equiv(1 / 2) \overline{u_{i} u_{i}} \\ R e_{\tau} & \text { Reynolds number in wall units } \\ U_{c} & \text { centerline velocity } \\ U_{i} & \text { instantaneous velocity } \\ U_{w} & \text { wall velocity } \\ u_{i} & \text { fluctuating velocity component } \\ u_{\tau} & \text { friction velocity } \\ n & \text { unit wall-normal vector } \\ t & \text { time } \\ x_{i} & \text { Cartesian directions, } i=1,2,3 \\ y_{w} & \text { wall-normal distance } \\ \delta(t) & \text { channel half-height at time } t \\ \partial_{i} & \text { spatial derivative in the } i \text { direction } \\ \nu & \text { kinematic viscosity } \\ & \end{array}$

*Postdoctoral fellow, AIAA member, elbert.jeyapaul@nasa.gov

$\dagger^{\dagger}$ Computational AeroSciences Branch, M.S. 128, g.n.coleman@nasa.gov

${ }^{\ddagger}$ Computational AeroSciences Branch, M.S. 128, AIAA Fellow, c.1.rumsey@nasa.gov 


$\begin{array}{ll}\text { Superscript } & \\ + & \text { wall units, scaled by } u_{\tau} \text { and } \nu \\ \text { Subscript } & \text { normalization by the respective r.m.s. } \\ \frac{i, j, k, l, m}{(\cdot)} & \text { indices of tensors } \\ & \text { Reynolds-averaged quantity }\end{array}$

\section{Introduction}

Second and higher-order Reynolds-averaged Navier-Stokes (RANS) closures are primarily motivated by the need to accurately predict complex flows. Convection-dominated flows, such as atmospheric boundary layers, have been shown to benefit from higher-order moment transport models; ${ }^{1}$ the third-order RANS model described in Hanjalic and Launder ${ }^{2}$ predicts the scalar flux accurately in a stably stratified flow, while the transport models used in second-moment closures (SMC) can capture the observed negative ('up-gradient') turbulent heat flux. ${ }^{3}$ The accurate prediction of the third-order moment transport model of Kurbatskii and Poroseva ${ }^{4}$ provides another example of the advantages of higher-order schemes. On the other hand, while higher-order closures are potentially more universal than their lower-order counterparts, they require more terms to be modeled and a larger set of coupled equations to be solved. The number of equations required increases rapidly as the dimensionality of the flow increases, as illustrated in figure 1 . This cost is compounded by numerical stiffness ${ }^{4}$ due to the difference in time-scales associated with moments of different orders.

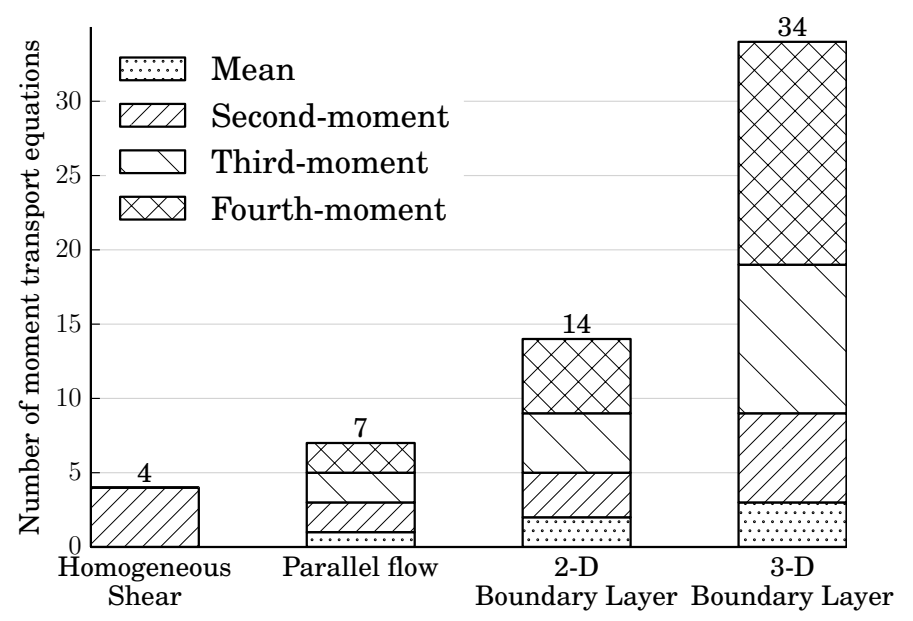

Figure 1. Minimum number of transport equations necessary for higher-order incompressible RANS closures of different flows. In practice, additional transport equations are often used to account for the dissipation terms.

In this paper we limit our attention to third- and fourth-order moments and the terms in their budgets (i.e., their transport equations), in an attempt to evaluate and improve the fidelity and universality of higherorder RANS closures. Beyond SMC, most of the research has been on truncation at third order, with an appeal to the Millionshtchikov hypothesis, ${ }^{5}$ which gives an algebraic closure for the fourth-order correlation as a product of quadratic moments. This hypothesis is exact for a Gaussian velocity distribution. As the bulk of most flows of interest are non-Gaussian, this approximation can be inaccurate. In the fourth-order-moment transport equations, models for the fourth-order velocity-pressure-gradient correlation and the dissipation are required, as are models for the fifth-order velocity correlations. In a recent hypothesis, ${ }^{6}$ fifth-order moments have been approximated by the sum of products of quadratic and cubic moments. This hypothesis assumes the probability distribution function to be a Gram-Charlier series, i.e., a truncated approximation about the Gaussian distribution. The Gram-Charlier approximation provides good a priori predictions in boundary layers. ${ }^{6}$ 
For simplicity, we analyze wall-bounded turbulence in a parallel-flow geometry. This idealization allows us to consider fewer equations than the full spatial case (figure 1). For parallel flows, like this one, the third-order RANS closure requires the solution of five velocity-momentum transport equations while seven are involved in a fourth-order scheme. At least one additional scale-setting equation, such as for dissipation, is also used in practice. Reference data consisting of results from the direct numerical simulation (DNS) of channel flow before and after it is subjected to the straining field introduced by an adverse pressure gradient $(\mathrm{APG})^{7,8}$ are used to perform a priori testing of existing third- and fourth-order models. This work is thus in the spirit of that presented by Yorke and Coleman ${ }^{9}$ and Sciberras and Coleman, ${ }^{10}$ who respectively performed a posteriori testing of scalar-eddy-viscosity and SMC schemes using this flow. Several new thirdand fourth-order models are also introduced in this work.

This paper is organized as follows. First, the strained-channel strategy used in the DNS is described, then the moment transport budget equations are provided. The DNS results are then summarized. Section IV includes both an overview and a discussion of higher-order modeling implications, where 'higher-order' refers to third- and fourth-order. A review of existing higher-order closure assumptions is then given, and several new modeling ideas are also presented. Finally, a priori model testing is conducted using the DNS results. By looking at the behavior of the various models in an a priori sense, we hope to gain some insight into which models might perform best when included in a complete model. It is recognized that a priori testing of model components has only limited practical value, because in many cases large terms almost balance each other and it is the combined effect of all the terms that matters. A posteriori testing of an entire scheme will ultimately be necessary to properly evaluate any chosen models.

\section{Strained-channel strategy}

Higher-order RANS closures are tested by comparing them with DNS of both conventional turbulent plane-channel flow and a strained-channel idealization of the APG boundary layer. The latter emulates the spatially developing low-Mach-number APG boundary layer by simultaneously applying in-plane 'sliding' motion of the walls and straining the domain of an incompressible turbulent channel flow, as shown in figure 2. The in-plane wall motion causes the bulk-flow deceleration needed to reduce the wall shear stress. The wall velocity $U_{w}(t)$ is coupled with the mean streamwise centerline velocity $U_{c}$, such that their difference decreases in time, according to $U_{c}-U_{w}=U_{c}(0) \exp \left(A_{11}\right)$, where $U_{c}(0)$ is the mean centerline velocity at $t=0$, when the strain is applied, and $A_{11}<0$ is the streamwise compression induced by the (virtual) APG. (Recall that in a parallel flow, wall motion is physically equivalent to a spatially uniform acceleration/deceleration.) Consequently, in the frame of reference attached to the walls, the history of the mean centerline velocity is given by $U_{c}(0) \exp \left(A_{11} t\right)$.

The (rate of) applied strain $A_{i j}$ is irrotational and uniform in space; it steps from zero at $t=0$ and is constant thereafter, which causes the entire domain (including the walls) to deform (figure 2). Here only the 'APG components' are involved, namely the streamwise deceleration $A_{11}<0$ and wall-normal divergence $A_{22}=-A_{11}>0$. For further details the reader is referred to Coleman et al. ${ }^{7}$
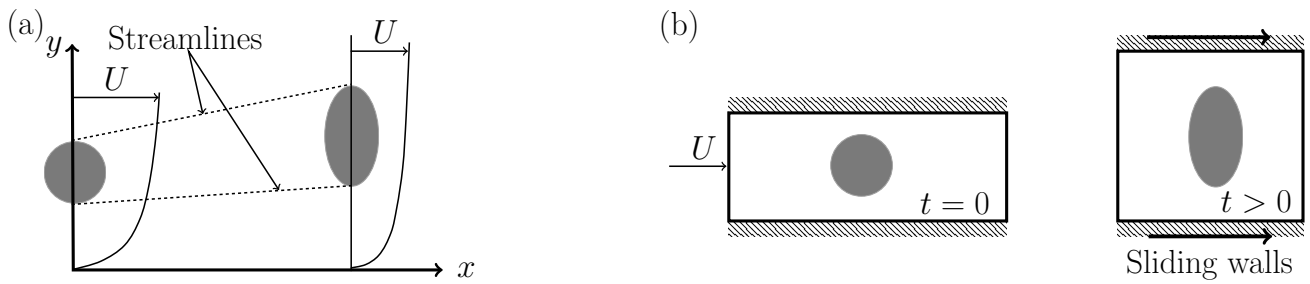

Figure 2. Turbulence in (a) a spatially developing APG boundary layer is simulated as (b) a time-evolving plane channel flow by combining the effects of irrotational strain that deforms the domain and sliding of the walls.

The strained-channel approach has the advantage of producing the desired perturbation in an uncomplicated parallel-flow geometry that contains essential features of the flow in question. Features of the APG boundary layer that are captured include the straining effect of the divergence of outer-layer streamlines and the reduction of wall shear stress. Missing is the effect of streamline curvature, which becomes important as the flow approaches the separation point. Also, pressure in an APG boundary layer experiences no 'reflection' effects from the inviscid outer region, whereas in the channel the pressure and its correlations are 


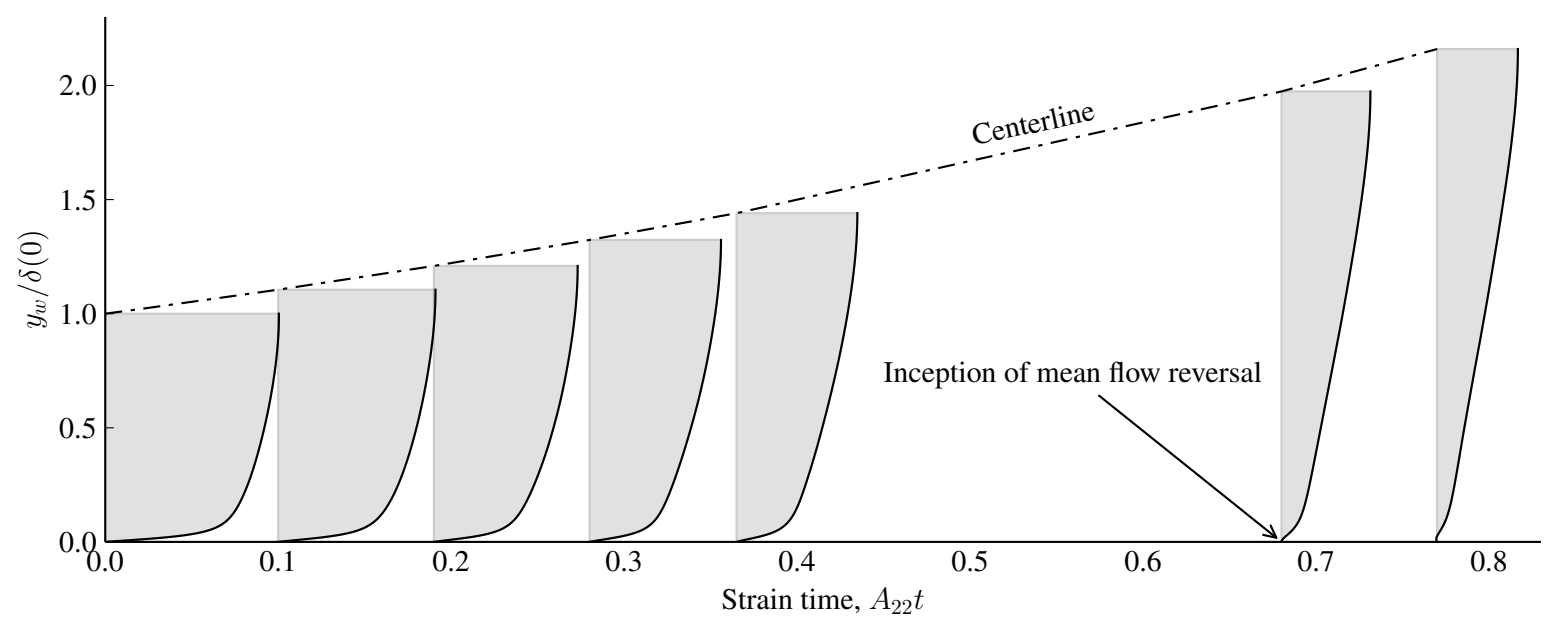

Figure 3. Spatially developing APG boundary layer represented by a temporally developing strained channel.

influenced by the opposite wall. These limitations are offset by the fact that the present approach reduces the RANS problem from a two-dimensional (2D) steady flow to a one-dimensional unsteady analog, which allows efficient model testing. This strategy has been previously used to assess eddy-viscosity ${ }^{9}$ and $\mathrm{SMC}^{10}$ models applied to the APG case. The reduction in dimensionality also greatly simplifies the higher-order RANS testing done here, since the parallel-flow assumption requires the solution of only half the number of velocity-moment equations required for the spatial counterpart (see figure 1).

\section{Moment transport budgets}

For the unsteady parallel-flow idealization of the APG boundary layer used here, for which the only nonzero mean spatial gradients are in the wall-normal direction $\left(x_{2}\right)$, the second-moment transport equation reduces to

$$
\partial_{t} \overline{u_{i} u_{j}}=\mathcal{P}_{i j}^{S}+\mathcal{P}_{i j}^{A}+\mathcal{T}_{i j}+\Pi_{i j}+\varepsilon_{i j}+\mathcal{D}_{i j}
$$

where

$$
\begin{aligned}
\mathcal{P}_{i j}^{S} & =-\overline{u_{i} u_{2}} \partial_{2} U_{j}-\overline{u_{j} u_{2}} \partial_{2} U_{i} \\
\mathcal{P}_{i j}^{A} & =-\overline{u_{i} u_{k}} A_{j k}-\overline{u_{j} u_{k}} A_{i k} \\
\mathcal{T}_{i j} & =-\partial_{2} \overline{u_{2} u_{i} u_{j}} \\
\Pi_{i j} & =-(1 / \rho)\left(\overline{u_{j} \partial_{i} p}+\overline{u_{i} \partial_{j} p}\right) \\
\varepsilon_{i j} & =-2 \nu \overline{\partial_{k} u_{i} \partial_{k} u_{j}} \\
\mathcal{D}_{i j} & =\nu \partial_{2}^{2} \overline{u_{i} u_{j}},
\end{aligned}
$$

where $\partial_{2}$ is the spatial derivative in the wall-normal direction. 
The third-moment transport equation is given by

$$
\partial_{t} \overline{u_{i} u_{j} u_{l}}=\mathcal{P}_{i j l}^{S}+\mathcal{P}_{i j l}^{A}+\mathcal{P}_{i j l}^{T}+\mathcal{T}_{i j l}+\Pi_{i j l}+\varepsilon_{i j l}+\mathcal{D}_{i j l},
$$

where

$$
\begin{aligned}
\mathcal{P}_{i j l}^{S} & =-\overline{u_{i} u_{j} u_{2}} \partial_{2} U_{l}-\overline{u_{j} u_{l} u_{2}} \partial_{2} U_{i}-\overline{u_{l} u_{i} u_{2}} \partial_{2} U_{j} \\
\mathcal{P}_{i j l}^{A} & =-\overline{u_{i} u_{j} u_{k}} A_{l k}-\overline{u_{j} u_{l} u_{k}} A_{i k}-\overline{u_{l} u_{i} u_{k}} A_{j k} \\
\mathcal{P}_{i j l}^{T} & =\overline{u_{i} u_{j}} \partial_{2} \overline{u_{2} u_{l}}+\overline{u_{j} u_{l}} \partial_{2} \overline{u_{2} u_{i}}+\overline{u_{l} u_{i}} \partial_{2} \overline{u_{2} u_{j}} \\
\mathcal{T}_{i j l} & =-\partial_{2} \overline{u_{2} u_{i} u_{j} u_{l}} \\
\Pi_{i j l} & =-(1 / \rho)\left(\overline{u_{i} u_{j} \partial_{l} p}+\overline{u_{j} u_{l} \partial_{i} p}+\overline{u_{l} u_{i} \partial_{j} p}\right) \\
\varepsilon_{i j l} & =-2 \nu\left(\overline{u_{i} \partial_{k} u_{j} \partial_{k} u_{l}}+\overline{u_{j} \partial_{k} u_{i} \partial_{k} u_{l}}+\overline{u_{l} \partial_{k} u_{i} \partial_{k} u_{j}}\right) \\
\mathcal{D}_{i j l} & =\nu \partial_{2}^{2} \overline{u_{i} u_{j} u_{l}},
\end{aligned}
$$

while the fourth-order moment counterpart is

$$
\partial_{t} \overline{u_{i} u_{j} u_{l} u_{m}}=\mathcal{P}_{i j l m}^{S}+\mathcal{P}_{i j l m}^{A}+\mathcal{P}_{i j l m}^{T}+\mathcal{T}_{i j l m}+\Pi_{i j l m}+\varepsilon_{i j l m}+\mathcal{D}_{i j l m},
$$

where

$$
\begin{aligned}
\mathcal{P}_{i j l m}^{S}= & -\overline{u_{i} u_{j} u_{l} u_{2}} \partial_{2} U_{m}-\overline{u_{j} u_{l} u_{m} u_{2}} \partial_{2} U_{i}-\overline{u_{l} u_{m} u_{i} u_{2}} \partial_{2} U_{j}-\overline{u_{m} u_{i} u_{j} u_{2}} \partial_{2} U_{l} \\
\mathcal{P}_{i j l m}^{A}= & -\overline{u_{i} u_{j} u_{l} u_{k}} A_{m k}-\overline{u_{j} u_{l} u_{m} u_{k}} A_{i k}-\overline{u_{l} u_{m} u_{i} u_{k}} A_{j k}-\overline{u_{m} u_{i} u_{j} u_{k}} A_{l k} \\
\mathcal{P}_{i j l m}^{T}= & \overline{u_{i} u_{j} u_{l}} \partial_{2} \overline{u_{2} u_{m}}+\overline{u_{j} u_{l} u_{m}} \partial_{2} \overline{u_{2} u_{i}}+\overline{u_{l} u_{m} u_{i}} \partial_{2} \overline{u_{2} u_{j}}+\overline{u_{m} u_{i} u_{j}} \partial_{2} \overline{u_{2} u_{l}} \\
\mathcal{T}_{i j l m}= & -\partial_{2} \overline{u_{2} u_{i} u_{j} u_{l} u_{m}} \\
\Pi_{i j l m}= & -(1 / \rho)\left(\overline{u_{i} u_{j} u_{l} \partial_{m} p}+\overline{u_{j} u_{l} u_{m} \partial_{i} p}+\overline{u_{l} u_{m} u_{i} \partial_{j} p}+\overline{u_{m} u_{i} u_{j} \partial_{l} p}\right) \\
\varepsilon_{i j l m}= & -2 \nu \overline{\left(u_{i} u_{j} \partial_{k} u_{l} \partial_{k} u_{m}\right.}+\overline{u_{i} u_{l} \partial_{k} u_{j} \partial_{k} u_{m}}+\overline{u_{j} u_{l} \partial_{k} u_{i} \partial_{k} u_{m}} \\
& \left.+\overline{u_{j} u_{m} \partial_{k} u_{l} \partial_{k} u_{i}}+\overline{u_{l} u_{m} \partial_{k} u_{j} \partial_{k} u_{i}}+\overline{u_{m} u_{i} \partial_{k} u_{l} \partial_{k} u_{j}}\right) \\
\mathcal{D}_{i j l m}= & \nu \partial_{2}^{2} \overline{u_{i} u_{j} u_{l} u_{m}} .
\end{aligned}
$$

For future reference, we note that the velocity-pressure-gradient correlations $\Pi_{i j}, \Pi_{i j l}$ and $\Pi_{i j l m}$ can each be decomposed into a term $(\phi)$ involving the product of pressure and gradients of velocity and another $(\psi)$ containing only spatial gradients of pressure-velocity correlations, such that $\Pi_{i j}=\phi_{i j}+\psi_{i j}, \Pi_{i j l}=\phi_{i j l}+\psi_{i j l}$ and $\Pi_{i j l m}=\phi_{i j l m}+\psi_{i j l m}$, where

$$
\begin{aligned}
\phi_{i j} & =(1 / \rho)\left(\overline{p \partial_{i} u_{j}}+\overline{p \partial_{j} u_{i}}\right), \quad(\text { i.e. the pressure-strain term }), \\
\phi_{i j l} & =(1 / \rho)\left(\overline{p \partial_{l} u_{i} u_{j}}+\overline{p \partial_{i} u_{j} u_{l}}+\overline{p \partial_{j} u_{l} u_{i}}\right), \\
\phi_{i j l m} & =(1 / \rho)\left(\overline{p \partial_{m} u_{i} u_{j} u_{l}}+\overline{p \partial_{i} u_{j} u_{l} u_{m}}+\overline{p \partial_{j} u_{l} u_{m} u_{i}}+\overline{p \partial_{l} u_{m} u_{i} u_{j}}\right), \\
\psi_{i j} & =(-1 / \rho)\left(\partial_{2} \overline{p u_{j}} \delta_{2 i}+\partial_{2} \overline{p u_{i}} \delta_{2 j}\right), \quad \text { (i.e. the pressure transport term), } \\
\psi_{i j l} & =(-1 / \rho)\left(\partial_{2} \overline{p u_{i} u_{j}} \delta_{2 l}+\partial_{2} \overline{p u_{j} u_{l}} \delta_{2 i}+\partial_{2} \overline{p u_{l} u_{i}} \delta_{2 j}\right), \\
\psi_{i j l m} & =(-1 / \rho)\left(\partial_{2} \overline{p u_{i} u_{j} u_{l}} \delta_{2 m}+\partial_{2} \overline{p u_{j} u_{l} u_{m}} \delta_{2 i}+\partial_{2} \overline{p u_{l} u_{m} u_{i}} \delta_{2 j}+\partial_{2} \overline{p u_{m} u_{i} u_{j}} \partial_{2 l}\right) .
\end{aligned}
$$

The terms on the right-hand side of (1)-(3) balance the total material derivative $D_{t}$. However, for the present parallel flows, the convection term is zero. With a constant streamwise pressure gradient and no applied strain, the statistics are steady, and $D_{t}=0$. When the flow is strained and the pressure gradient is replaced by the effect of the in-plane wall motion, the material derivative is $D_{t}=\partial_{t} \neq 0$, and the moments evolve in time, analogous to the spatial development of an adverse pressure gradient boundary layer. ${ }^{7}$ In other words, the streamwise spatial derivatives of boundary layers are replaced here by time derivatives.

In equations (1)-(3), terms on the right-hand side are referred to as (nominal) production due to shear $\mathcal{P}^{S}$, production due to Reynolds stress $\mathcal{P}^{T}$, turbulent transport $\mathcal{T}$, velocity-pressure-gradient correlation $\Pi$, dissipation $\varepsilon$, and viscous diffusion $\mathcal{D}$. The applied strain produces non-zero terms in all the moment equations, termed production due to applied strain $\mathcal{P}^{A}$. The terminologies used to describe the terms in the third- and fourth-order budgets are inherited from second-moment closures, and do not necessarily describe their physical behavior. That is, 'production' can be negative and 'dissipation' can be positive in the thirdand fourth-order moment equations. 


\section{DNS results}

\section{A. Overview}

The DNS is performed by a pseudo-spectral (Fourier/Chebyshev- $\tau$ ) method. The parameters and procedures of this method are described in Coleman et al. ${ }^{7}$ The simulation was repeated for this study because the third, fourth- and fifth-order moments of interest here were not part of the original study. The new statistics were evaluated from a new ensemble of 159 fields (in each field, averaging was also performed over streamwisespanwise planes and folded about the centerline, taking advantage of the symmetry), using $256 \times 193 \times 192$ spectral modes in a $2 \pi \delta(0) \times \delta(0) \times \pi \delta(0)$ domain (where $\delta(0)$ is the half-height of the initial/pre-strained channel), in the streamwise $x=x_{1}$, wall-normal $y=x_{2}$ and spanwise $z=x_{3}$ directions, respectively. The runs were made on Pleaides, an SGI ${ }^{\circledR}$ ICE-X cluster using Intel Xeon processors at the NASA Advanced Supercomputing division. The unstrained channel simulations required 11,000 processing core hours to obtain the 159 independent realizations, which were used as initial conditions for the corresponding 159 strained-channel runs, requiring a further $8,000(\approx 50 \times 159)$ hours to advance each of them to a strain time of $A_{22} t=0.77$ (well past the point of separation; see below).

The fully-developed (unstrained) channel Reynolds number $R e_{\tau}=u_{\tau} \delta / \nu$ is 392, which is large enough to sustain a well-defined inertial sublayer. At this initial unstrained state $\left(A_{22} t=0\right)$, the Reynolds number based on the mean centerline velocity $R e_{c} \equiv U_{c} \delta / \nu$ is 7,910, while the momentum-thickness and bulk Reynolds numbers are respectively $R e_{\theta} \equiv U_{c} \theta / \nu=703$ and $R e_{m} \equiv 2 \delta U_{m} / \nu=13,770$ (where $\theta$ is the halfchannel momentum thickness, and $U_{m}$ the bulk, wall-to-wall, average velocity). As in Ref. 7, a relatively weak APG is applied to the series of realizations of unstrained channel-flow turbulence, with $A_{22}=-A_{11}$ chosen to be $31 \%$ of $u_{\tau}(0) / \delta(0)$, the ratio of the initial friction velocity to the initial channel half-width. The applied strain $\left|A_{11}\right|$ is at least an order of magnitude smaller than the mean shear $\partial U / \partial y$ over the entire channel for all strain times. ${ }^{9}$ Consequently, the magnitude of the applied irrotational strain is not large enough at any channel height to cause the non-linear interactions of turbulence to be negligible; it is these nonlinear interactions that the higher-order RANS models are designed to predict with higher fidelity than SMCs. Both the strain-dependent 'fast' and strain-independent 'slow' parts of the budget terms are thus expected to contribute to the moment evolution.

The resulting mean velocity profiles are shown in figure 3. Many of the basic features of APG boundary layers are present. For example, the shape factor (in terms of the displacement and momentum thickness in the half-channel) increases from $H=\delta^{*} / \theta=1.45$ at $A_{22} t=0$ to $H=1.7$ at $A_{22} t=0.365$ and $H=2.5$ at $A_{22} t=0.77$ - past the time at which the mean 'separation' (i.e. mean-flow reversal at the wall) occurs, at $A_{22} t=0.68$. This is consistent with an axisymmetric-body separation-bubble, ${ }^{7}$ for which $H \approx 2.7$. The effective Clauser parameter $\beta_{\text {eff }}=-\delta^{*} U_{c} A_{11} / u_{\tau}^{2}$ is 0.78 at $A_{22} t=0$, before increasing to 5.7 at $A_{22} t=0.365$.

\section{B. Higher-order-modeling implications}

Normalized statistics such as skewness and flatness of velocity correlations vary through the channel height (figure 4). Higher-order models will need to resolve this variation by using models for unknown terms to close equations (2) and (3). The unstrained-channel skewness and flatness profiles compare well with prior channel-flow DNS. ${ }^{12}$ Experimental studies of a turbulent boundary layer ${ }^{11}$ show the skewness and flatness reach their global maxima or minima near the wall and the boundary-layer edge, with a nearly constant value between the two extrema. This trend is not noticed in the channel-flow skewness (figure 4a), in that here both $S_{1}$ and $S_{2}$ do not have their maxima/minima near the wall nor at the channel centerline. In the inertial subregion, the APG strain (until $A_{22} t=0.365$ ) drives the skewness for all three velocity components toward zero (i.e. toward the Gaussian state). The strain also causes the flatness for the wall-normal $\left(F_{2}\right)$ and spanwise $\left(F_{3}\right)$ velocities to reduce throughout the channel toward the Gaussian value of 3, and for the streamwise component to become slightly less Gaussian than it was before the strain is applied, reducing from $F_{1} \approx 3$ to about 2.7. The effect of the straining in this region is similar to that seen for the mild-APG boundary layer ${ }^{11}$ (the skewness and flatness differences observed in the very-near-wall and wake regions are presumably related to near-wall measurement difficulties and/or to the difference between the channel and boundary-layer geometries).

For the present flow, the moments that act as dependent variables in a fourth-order RANS closure are $-\overline{u v}, \overline{v^{2}}, \overline{u v^{2}}, \overline{v^{3}}, \overline{u v^{3}}$ and $\overline{v^{4}}$. (Models for the pressure-velocity correlations and dissipation could in principal introduce dependence on additional moments, but we do not consider that possibility here.) 

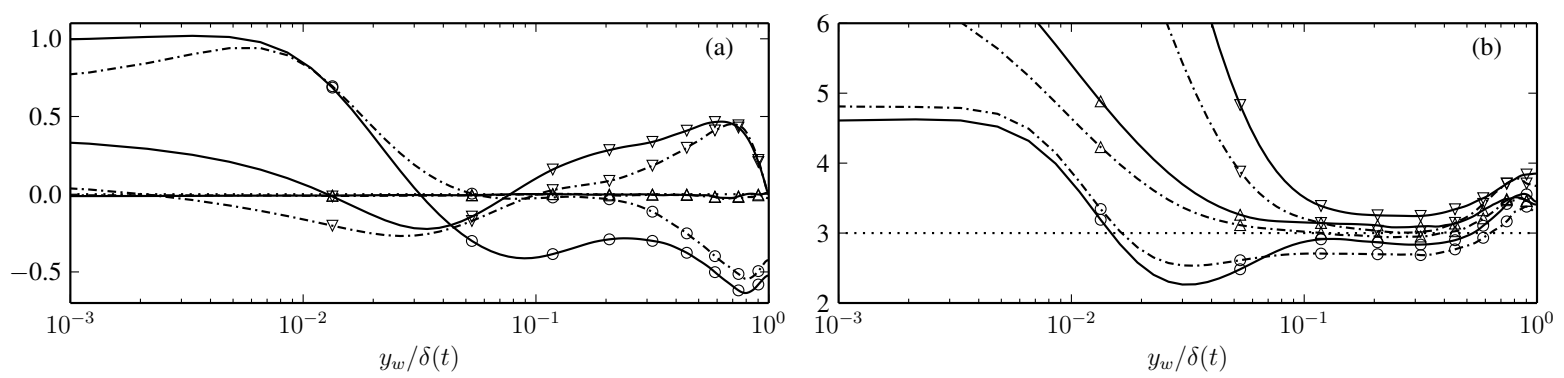

Figure 4. Plots of (a) skewness $S_{\alpha}=\overline{u_{\alpha}^{3}} /\left(\overline{u_{\alpha}^{2}}\right)^{3 / 2}$ and (b) flatness $F_{\alpha}=\overline{u_{\alpha}^{4}} /\left(\overline{u_{\alpha}^{2}}\right)^{2}$ (no sum over $\alpha=1,2,3$ ): $A_{22} t=0 ;-\cdot-, A_{22} t=0.365 ; \circ, u_{1}=u ; \nabla, u_{2}=v$ and $\Delta, u_{3}=w$.

The evolution of the relevant third- and fourth-order moments and the terms in their budget are shown in figures 5-8 for the unstrained case $A_{22} t=0$ and for the strained case at $A_{22} t=0.365$. The smooth nature of the profiles in figure 4 indicates that the statistical error is fairly low. For the unstrained flow, checks were performed to ensure that these statistical errors are negligible, by comfirming that the sum of the right-handside budget terms from the 159-field ensemble is much smaller than the smallest term in each budget. It was also verified that the third- and fourth-order moments were not corrupted by aliasing errors, by recomputing them after projecting the velocity fields onto a collocation grid that is $2^{3}$ times larger, and confirming that no significant differences result, compared to the moments obtained from forming the products on the grid used for the DNS. Moreover, the sum of the right-hand-side terms in equations (2) and (3) are very close to the observed time rate of change of the moment being transported (figure not shown), which gives further confidence in the DNS and the calculations of the terms in each of the higher-moment budgets.

The third-order moments, which measure the skewness of the corresponding probability distribution function (PDF), are smaller than the second- and fourth-order moments. The third-order moments $\overline{v^{3}}$ and $-\overline{u v^{2}}$ have inflectional profiles, with negative values near the wall and positive values elsewhere (figures 5 and 6 respectively). The left-hand-side subplot in each of these composite figures shows the moment evolution and, on the right-hand side, the strain-induced changes to each of the terms in its budget, equation (2). We notice negative $\overline{v^{3}}$ values at $y_{w} / \delta<0.1$, which is primarily caused by an imbalance between $\Pi_{222}, \mathcal{P}_{222}^{T}$ and $\mathcal{T}_{222}$. Both $\Pi$ and $\mathcal{T}$ are unclosed terms, needing modeling. Note that $\mathcal{P}_{222}^{S}=0$, hence $\overline{v^{3}}$ is not affected directly by the mean velocity. The direct effects of the applied irrotational straining are manifested through $\mathcal{P}_{222}^{A}$. The strain applied at $A_{22} t=0$ causes an instantaneous increase in both the velocity-pressure-gradient term (due to incompressibility) and the additional negative 'production' $\mathcal{P}^{A}$. As seen in inset figure $5 \mathrm{~d}$, $\mathcal{P}_{222}^{A}$ dominates $\Pi_{222}$, causing $\overline{v^{3}}$ to increase throughout the channel instantaneously. As noted in Coleman et al., ${ }^{7}$ this sudden increase is due to the channel turbulence not receiving a 'warning' of the impending discontinuous temporal change. This behavior is thus unlike a spatially developing boundary layer where convective changes in the mean flow propagate upstream. However, at $A_{22} t=0.365$, the difference between the inner- and outer-layer dynamics causes $\overline{v^{3}}$ to decrease in the inner layer and grow in the outer layer, similar to the Reynolds stresses. ${ }^{7}$ Notice (compare inset (c) to inset (d) of figure 5) that $\partial \overline{v^{3}} / \partial t$ is of the same order of magnitude at $A_{22} t=0$ and 0.365 . This implies that the step increase of $A_{11}=A_{22}<0$ at 0 does not derail the turbulence mechanisms, allowing the nonlinearities (manifested through change in budget terms) to take effect at later times, causing the different inner-outer layer dynamics. The inner-layer reduction and outer-layer growth of $\overline{v^{3}}$ are also seen past flow reversal $\left(A_{22} t=0.77\right)$.

The evolution of $\overline{u v^{2}}$ is caused by active contributions from nearly all the budget terms (figure 6). An inner-layer decrease and outer-layer growth is noticed, similar to that for $\overline{v^{3}}$. The direct effect of straining through $\mathcal{P}_{122}^{A}$ has a lesser role in $\partial \overline{u v^{2}} / \partial t$ than the net contribution (indirectly affected by straining) from the rest of the budget terms.

The fourth-order moments, which are all positive definite, show noticeably larger outer-layer increases than inner-layer decrease (figures 7 and 8). This difference between inner- and outer-layer response is thus different from that for the third-order moments. Interestingly, we notice $\overline{v^{4}}$ responds to strain in a manner similar to $\overline{v^{2}}$, although an additional term $\left(\mathcal{P}^{T}\right)$ contributes. The $\overline{v^{4}}$ budget is driven primarily by an imbalance between $\Pi_{2222}, \varepsilon_{2222}$ and $\mathcal{T}_{2222}$ (figure 7 ). The same budget terms contribute to the evolution of $\overline{v^{2}}$ (figure 9 in Ref. 7 ). The wall-normal flatness $F_{2}$ increases rapidly near the wall (figure 4 ) due to the differences in the rates of decay of the dominant budget terms, $\Pi, \varepsilon$ and $\mathcal{T}$, of which $\Pi_{2222}$ and $\varepsilon_{2222}$ remain 

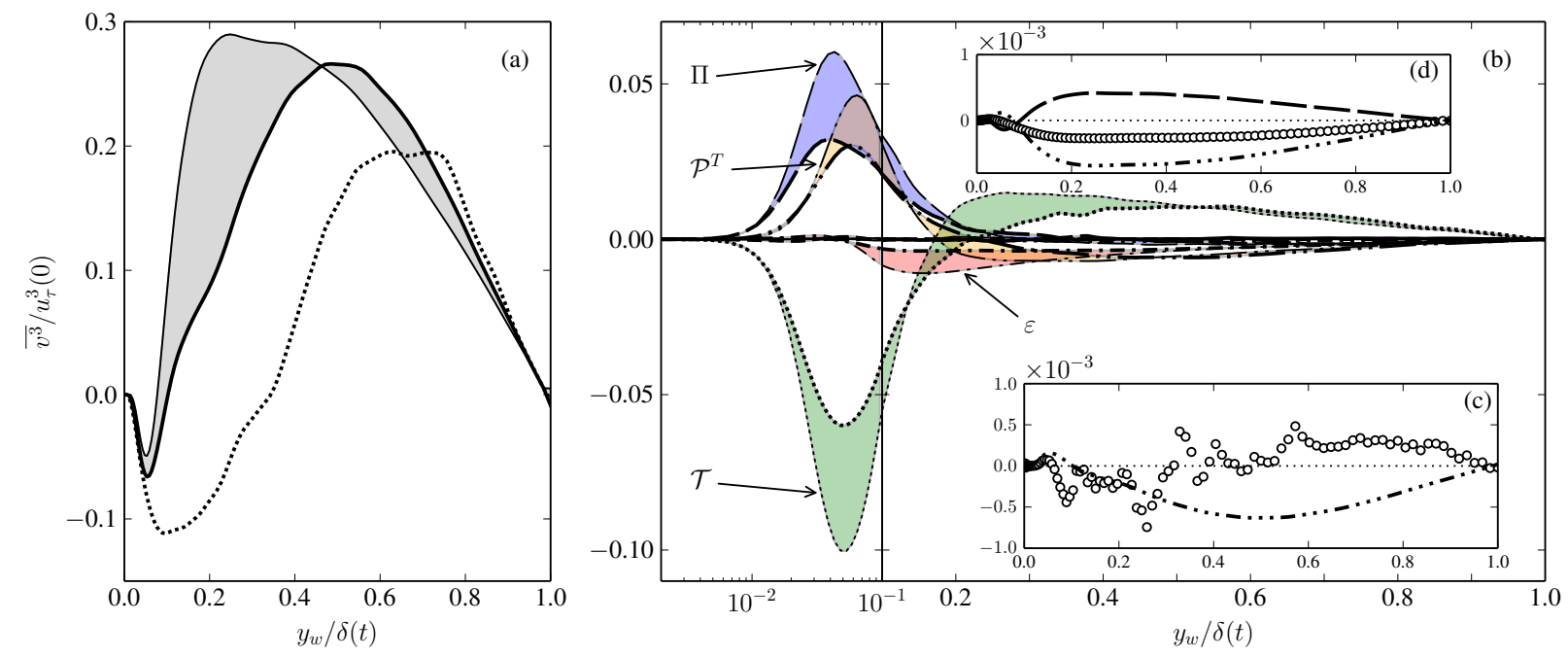

Figure 5. (a) Third-order wall-normal velocity correlation $\left(\overline{v^{3}}\right)$ profiles:,$- A_{22} t=0$;,$- A_{22} t=$ $0.365 ; \cdots \cdots, A_{22} t=0.77$. (b) Terms in the $\overline{v^{3}}$ budget at $A_{22} t=0.365:-\cdots-\cdots-$, Production due to shear $\left(\mathcal{P}^{S}\right) ; \quad-\cdots-, \quad$ Production due to Reynolds stress $\quad\left(\mathcal{P}^{T}\right) ; \quad$.............., Turbulent transport $\quad(\mathcal{T}) ; \quad---$, Velocity--pressure-gradient $(\Pi) ;-\cdot-\cdot--$, Dissipation $(\varepsilon) ;-\cdots-\cdots$, Production due to applied strain $\left(\mathcal{P}^{A}\right)$, also shown in insets $(c)$ and $(d) ;-----$, Viscous diffusion $(\mathcal{D})$. Thin curves denote terms at $\mathbf{t}=0$ (before strain) and are identified by the shaded regions, which indicate change from unstrained initial conditions. The budget terms in (b) are normalized by $u_{\tau}^{5}(0) / \nu$. Inset (c) Hollow circles (O) are sum of all budget terms $\left(\approx \partial \overline{v^{3}} / \partial t\right)$ at $A_{22} t=\mathbf{0 . 3 6 5}$. (d) Budget term balance due to instantaneous application of strain $\left(A_{22} t=0^{+}\right)$. Curves shown are subtracted from initial unstrained profile $\left(A_{22} t=0\right)$. The curve and symbol types are as in (b) and (c). The axes in insets (d) and (c) are non-dimensionalized as (b).
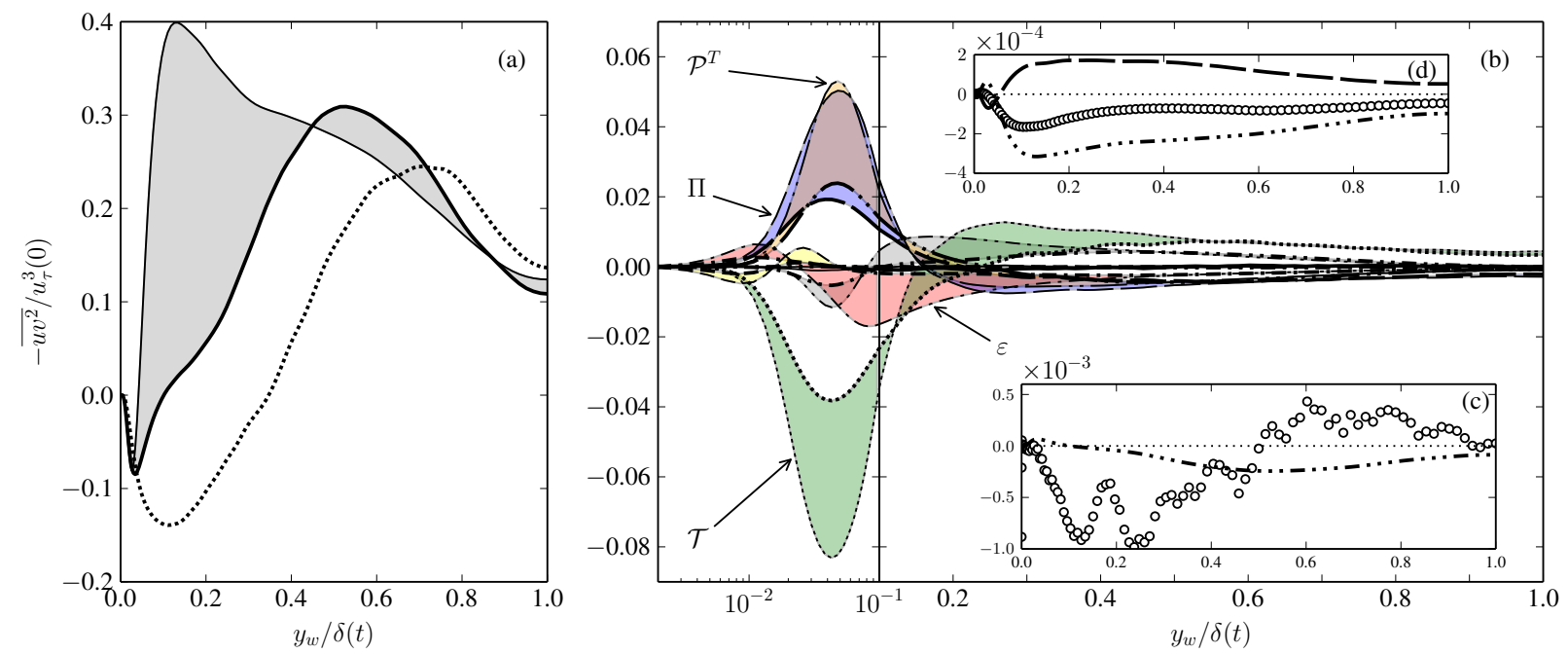

Figure 6. (a) Third-order shear-stress correlation $\left(-\overline{u v^{2}}\right)$ profiles:,$- A_{22} t=0 ;-, A_{22} t=0.365 ; \cdots \cdots$, $A_{22} t=0.77$. (b) Terms in the $\overline{u v^{2}}$ budget at $A_{22} t=0.365$. Inset (c) Hollow circles (O) are sum of all budget terms $\left(\approx \partial \overline{u v^{2}} / \partial t\right)$ at $A_{22} t=\mathbf{0 . 3 6 5}$. Inset $(\mathrm{d})$ budget term balance due to instantaneous application of strain $\left(A_{22} t=0^{+}\right)$. The budget terms are normalized by $u_{\tau}^{5}(0) / \nu$. For line legend and shading key refer to figure 5 . 

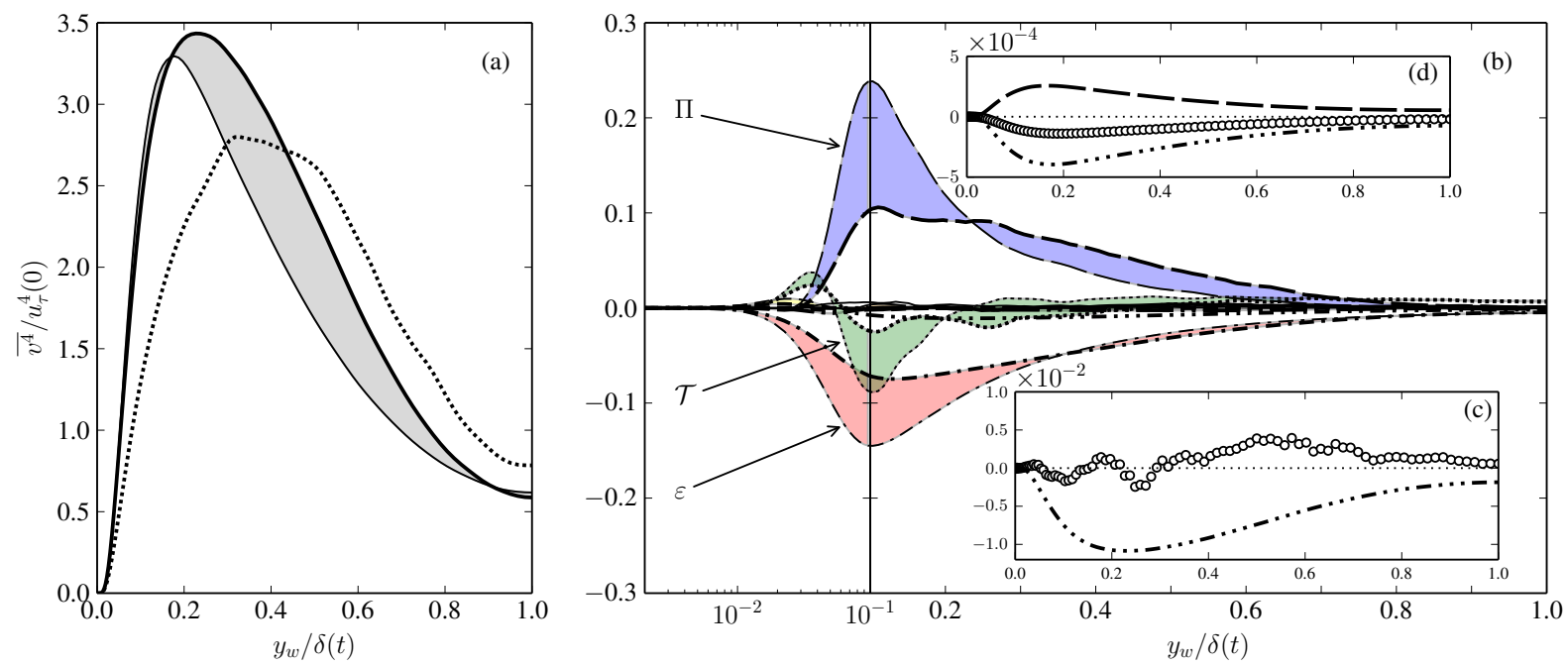

Figure 7. (a) Fourth-order wall-normal velocity correlation $\left(\overline{v^{4}}\right)$ profiles:,$- A_{22} t=\mathbf{0} ;-, A_{22} t=\mathbf{0 . 3 6 5}$; $\ldots \ldots, A_{22} t=0.77$. (b) Terms in the $\overline{v^{4}}$ budget at $A_{22} t=\mathbf{0 . 3 6 5}$. Inset (c) Hollow circles (O) are sum of all budget terms $\left(\approx \partial \overline{v^{4}} / \partial t\right)$ at $A_{22} t=\mathbf{0 . 3 6 5}$. Inset $(\mathrm{d})$ budget term balance due to instantaneous application of strain $\left(A_{22} t=0^{+}\right)$. The budget terms are normalized by $u_{\tau}^{6}(0) / \nu$. For line legend and shading key refer to figure 5 .
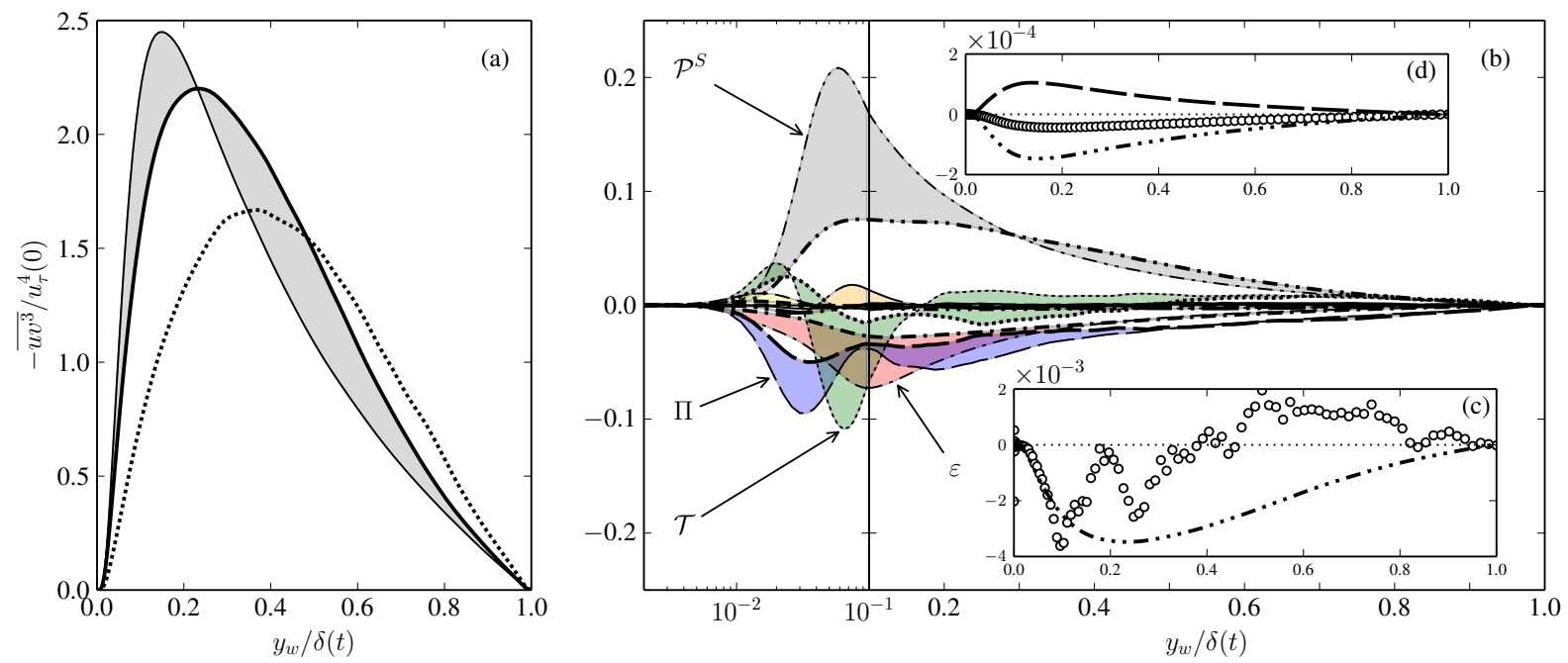

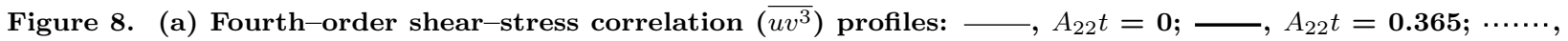
$A_{22} t=0.77$. (b) Terms in the $\overline{u v^{3}}$ budget at $A_{22} t=0.365$. Inset (c) Hollow circles (O) are sum of all budget terms $\left(\approx \partial \overline{u v^{3}} / \partial t\right)$ at $A_{22} t=\mathbf{0 . 3 6 5}$. Inset $(\mathbf{d})$ budget term balance due to instantaneous application of strain $\left(A_{22} t=0^{+}\right)$. The budget terms are normalized by $u_{\tau}^{6}(0) / \nu$. For line legend and shading key refer to figure 5 . 
the largest, since they decay slowest as $y_{w} \rightarrow 0$, being $\mathcal{O}\left(y_{w}^{6}\right)$ (see Appendix). It is therefore important that models for $\Pi_{2222}$ and $\varepsilon_{2222}$ reproduce this near-wall asymptotic behavior. The direct effect of straining through the applied production $\left(\mathcal{P}_{2222}^{A}=-4 \overline{v v v v} A_{22}\right)$ nearly balances the net effect of the other three dominant terms mentioned earlier (figure 7 ), causing $\overline{v^{4}}$ to grow for $y_{w} / \delta>0.3$ and decay near the wall. The centerline $\overline{v^{4}}$ decreases between $A_{22} t=0$ and 0.365 and increases from $A_{22} t=0.365$ to 0.77 , as the flow slowly recovers from the abrupt initial strain.

From the $\overline{u v^{3}}$ budgets in figure 8 we notice all the budget terms make a significant contribution to $\partial \overline{u v^{3}} / \partial t$. Production due to mean shear is a dominant term in the balance. Production due to turbulence $\left(\mathcal{P}_{1222}^{T}\right)$, although small, couples the $\overline{u v^{3}}$ evolution to the third-order moments (a route for non-Gaussian effects to manifest themselves). In the near-wall region $\left(y_{w} / \delta<0.1\right)$ the evolution of $\overline{u v^{3}}$ is primarily driven by $\mathcal{P}_{1222}^{A}$.

Since Rotta, ${ }^{13}$ it has become conventional in SMC to split the velocity-pressure-gradient term $\left(\Pi_{i j}\right)$ into the sum of pressure-strain $\phi_{i j}$ and pressure-transport (often referred to as 'pressure diffusion') $\psi_{i j}$ terms (see earlier equations (4) and (7)). For modelers this is advantageous, since pressure-strain is redistributive/tracefree (i.e., $-\phi_{11}=\phi_{22}+\phi_{33}$ ), and is thus responsible for transfer of energy between the three components, and pressure-transport is non-zero only near the wall. Therefore, in homogeneous turbulence, $\Pi_{i j}=\phi_{i j}$, making the problem amenable to the use of homogeneous turbulence theory to develop models. By definition, in homogeneous flows, $\psi_{i j l}=\psi_{i j l m}=0$, hence homogeneous theory can be extended to model the higher-order counterparts of $\phi_{i j}$. On the other hand, the wall boundary conditions on $\phi_{i j}$ and $\psi_{i j}$ are both non-zero, in fact they are equal and opposite, and therefore more complicated than the zero condition on each of the elements of $\Pi_{i j}$. This difficulty is a symptom of the larger problem that in the near-wall region, the magnitudes of $\phi_{i j}$ and $\psi_{i j}$ are much larger than their net effect, which will tend to amplify any errors in their respective models.

Some modelers ${ }^{3,14}$ have applied the $\Pi$ splitting to third-order budgets. In contrast to the second-order case, $\phi=0$ and $\psi=0$ at $y_{w}=0$ for both third and fourth order, and the fourth-order 'pressure-strain' is not redistributive, in the sense that $\phi_{i j i j}=(2 / \rho)\left(\overline{p \partial_{j} u_{i} u_{j} u_{j}}+\overline{p \partial_{i} u_{i} u_{j} u_{j}}\right) \neq 0$. Furthermore, as seen in the DNS results in figures 9 and 10, the magnitudes of $\phi_{i l l}$ and $\psi_{i l l}$, and of $\phi_{i j l m}$ and $\psi_{i j l m}$, are comparable in regions well away from the wall (although the APG strain does tend to drive $\psi_{i j l m} \approx 0$ there); we note that of the components considered here, only $\psi_{2222}$ is nearly zero and $\Pi_{2222} \approx \phi_{2222}$ in the outer-layer. And perhaps most importantly, from a modeling point of view, the wall-normal variations of $\Pi_{i l l}$ and $\Pi_{i j l m}$ are simpler then that of their constituent $\phi$ and $\psi$. All of this suggests there is little if any inherent advantage to the $\Pi=\phi+\psi$ decomposition for the higher-order moments.
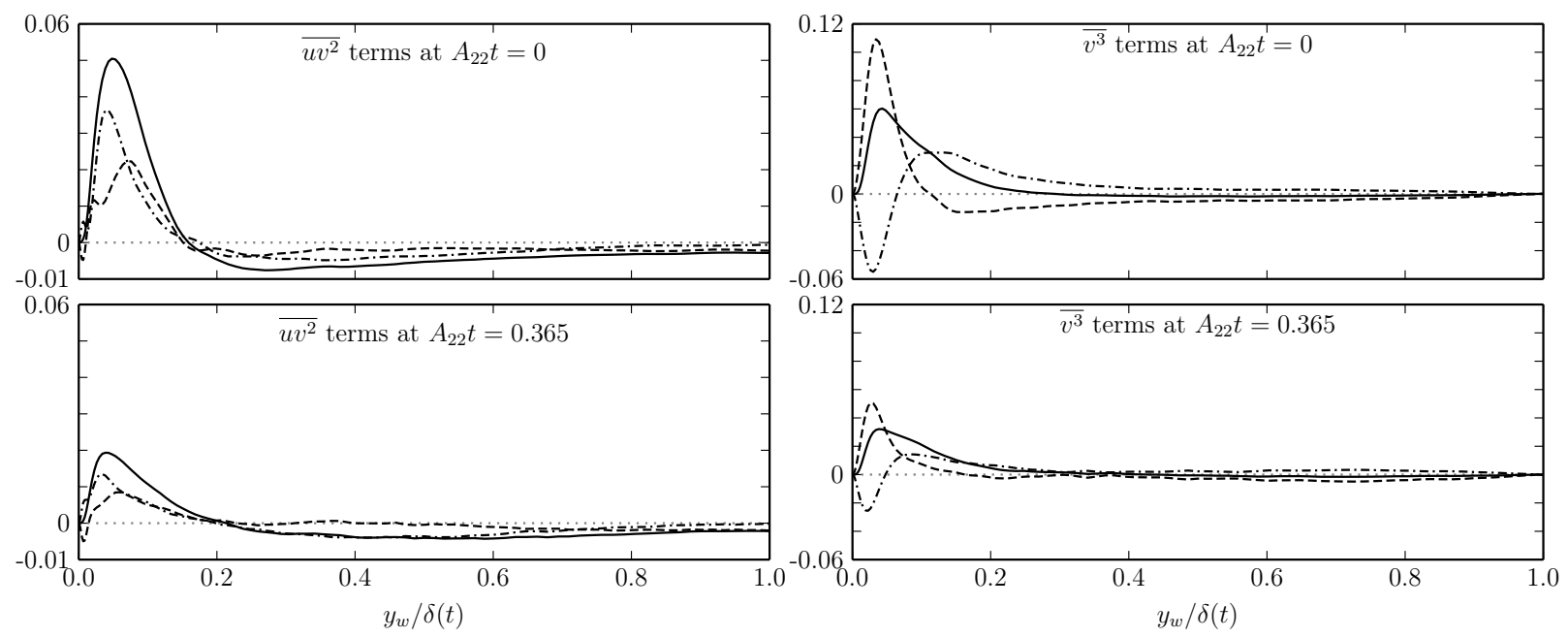

Figure 9. Third-order velocity-pressure-gradient budget term split before and after straining. The velocitypressure-gradient term $\Pi$ is $\longrightarrow$; pressure-strain term $\phi$ is -...-; pressure transport term $\psi$ is - - -..

Models for fourth-order moment evolution have been the least explored. Single-point fourth-order moments have major symmetries $\left(\overline{u_{i} u_{j} u_{l} u_{m}}=\overline{u_{j} u_{l} u_{m} u_{i}}=\overline{u_{l} u_{m} u_{i} u_{j}}=\overline{u_{m} u_{i} u_{j} u_{l}}\right)$ by definition. This reduces the number of independent components from $81\left(=3^{4}\right)$ to 21 and the number of principal invariants from 

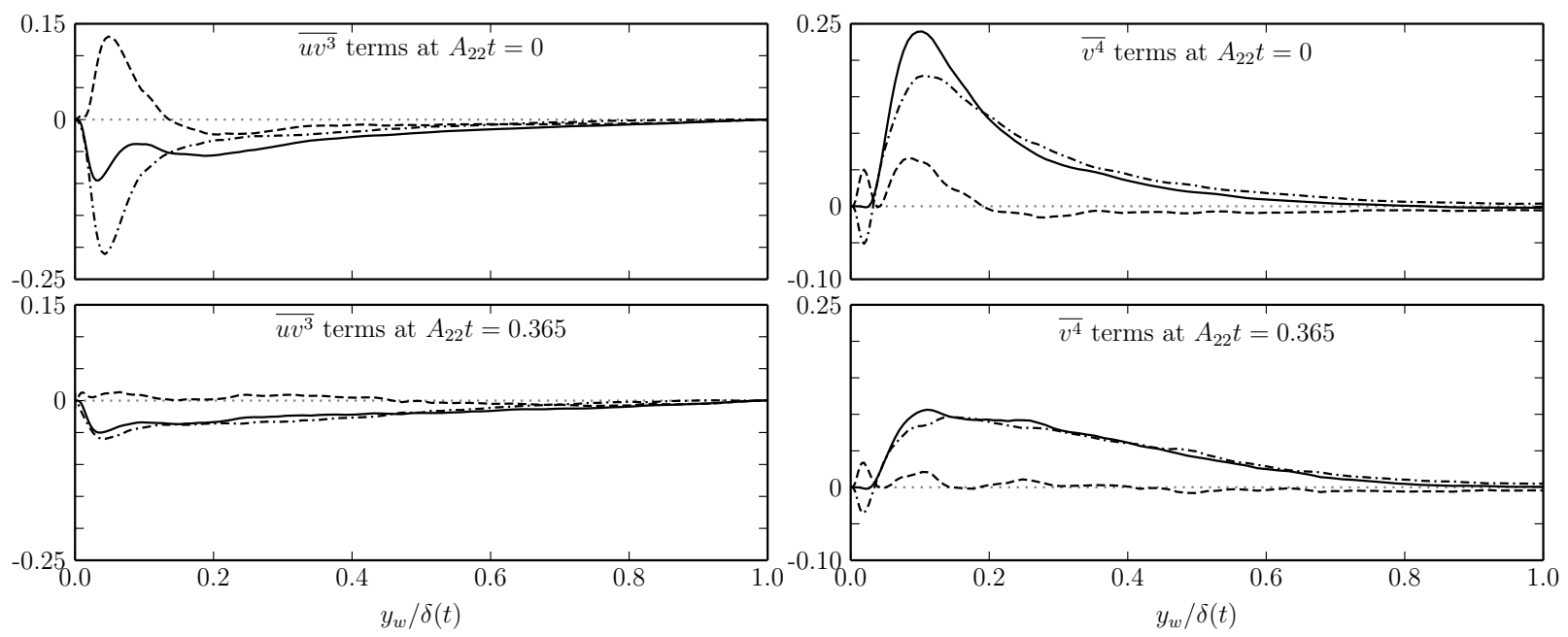

Figure 10. Fourth-order velocity-pressure-gradient budget term split before and after straining. For the line legend refer to figure 9 .

9 to 6 (see Batten ${ }^{15}$ ). The invariants are objective properties of the tensor, as they do not change with coordinate frame rotation. The simplest of invariants, the first, is the trace of the tensor, which for the fourth-order moment is $\operatorname{tr}\left(\overline{u_{i} u_{j} u_{l} u_{m}}\right)=\overline{u_{i} u_{j} u_{i} u_{j}}=\overline{u^{4}}+\overline{v^{4}}+\overline{w^{4}}+2\left(\overline{u^{2} v^{2}}+\overline{v^{2} w^{2}}+\overline{w^{2} u^{2}}\right)$. Turbulent kinetic energy is half the trace of the Reynolds stress tensor $K_{2} \equiv(1 / 2) \overline{u_{i} u_{i}}$. Similarly, a fourth-order analog of turbulent kinetic energy can be defined as $K_{4} \equiv(1 / 2) \overline{u_{i} u_{j} u_{i} u_{j}}$.

The quantity $K_{4}$ is positive definite and is a measure of the magnitude of the fourth-order moment. Its budget terms are descriptive of the terms they represent, that is, 'production' is positive definite and 'dissipation' is negative definite (figure 11). We notice from figure 11(a) that as a result of straining, $K_{4}$ is reduced drastically in the inner layer and increases in the outer layer of the channel, similar to turbulent kinetic energy $K_{2}$ (figure 7 of Ref. 7 ). As for $K_{2}$, for which the balance of budget terms is $\varepsilon_{k} \approx \mathcal{D}_{k}$ as $y_{w} \rightarrow 0$, the dissipation versus viscous-diffusion balance is also observed for $K_{4}$. However, in the $K_{2}$ budget, the dissipation and viscous diffusion are non-zero at the wall. We also notice that $\partial_{t} K_{4}$ evolves similar to $\partial_{t} K_{2}$ (compare figure 11(d) to figure 7 of Ref. 7). Evolution of $K_{4}$ in the outer half-channel is driven by applied-strain production $\left(=4\left(\overline{u^{4}}+\overline{v^{4}}+\overline{u^{2} w^{2}}-\overline{v^{2} w^{2}}\right) A_{22}\right)$, causing $K_{4}$ to steadily increase in the outer layer (figure 11(a)). The equivalent of a second-order turbulence time scale $\left(\tau_{2}=2 K_{2} /\left|\epsilon_{i i}\right|\right)$ ), a measure of the rate at which the turbulence kinetic energy is dissipated at the smallest scales, can also be defined for the fourth-order moment as $\tau_{4}=2 K_{4} /\left|\epsilon_{l m l m}\right|$. A plot of the ratios of these time scales (figure 11(c)) shows that $\tau_{2} \approx 2 \tau_{4}$ for both the unstrained and strained cases throughout most of the channel, except near the wall. That is, fourth-order moments tend to respond on the order of twice as fast as second-order moments. Scale-similarity models (see below) for the fourth-order budget terms need to account for this more rapid evolution. A simple model relating the fourth-order timescale to second-order timescale can be given by

$$
\tau_{4}=\frac{\tau_{2}}{f_{w}} ; \quad f_{w}=2+4 \exp \left[-\left(\frac{y^{+}(t)}{6}\right)^{2}\right],
$$

where, $y^{+}(t)=y^{+}(0) \delta(0) / \delta(t)$ is the wall-distance scaled by the initial unstrained channel height. The timescale ratio $f_{w}$ is only a function of wall distance, whose variation has been chosen to approximately reproduce the near-wall behavior of the DNS.

\section{Closure assumptions}

In this section, we introduce currently available models utilized in third- and fourth-moment transport RANS schemes. These modeling assumptions are summarized in Table 1. We also introduce several new models (using the nomenclature JCR1-6) in this section. Most of the models in Table 1, as well as the new 

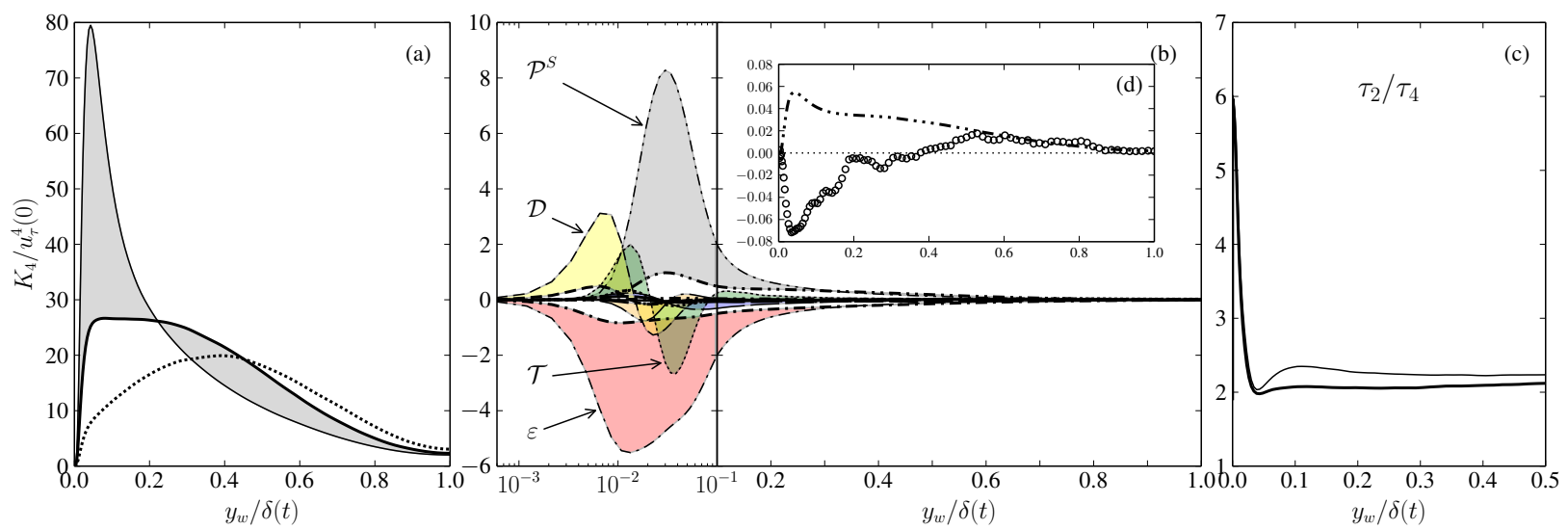

Figure 11. (a) Fourth-order turbulent kinetic energy analog $\left(K_{4} \equiv \overline{u_{i} u_{j} u_{i} u_{j}}\right)$ profiles at strain times: $A_{22} t=0 ;-, A_{22} t=0.365 ; \cdots \cdots, A_{22} t=0.77$. (b) Terms in the $K_{4}$ budget at $A_{22} t=0.365$. The budget terms are normalized by $u_{\tau}^{5}(0) / \nu$. For line legend and shading key refer to figure 5. (c) Ratio of second- and fourth-order turbulent time scales $\left(\tau_{2} / \tau_{4}\right)$, where $\tau_{4} \equiv \overline{u_{i} u_{j} u_{i} u_{j}} / \varepsilon_{l m l m}$ at strain times:,$- A_{22} t=0$; - , $A_{22} t=\mathbf{0 . 3 6 5}$.

JCR models, will be compared to the DNS data in Section VI.

Table 1. Existing models for terms in higher-order RANS.

\begin{tabular}{clcc}
\hline Moment-order & $\mathcal{T}$ & $\Pi$ & $\varepsilon$ \\
\hline $3^{\text {rd }}$ & $\mathrm{M}^{5}, \mathrm{KSK}^{16}, \mathrm{CCH}^{17}{ }^{17} \mathrm{GHRS}^{18}$, & $\mathrm{DL} 1,{ }^{3} \mathrm{NT},{ }^{14} \mathrm{KP} 1,{ }^{4} \mathrm{KSK}^{16}$ & $\mathrm{~A}^{1}, \mathrm{DL} 2,3^{3}, \mathrm{KSK}^{16}$ \\
$4^{\text {th }}$ & $\mathrm{GN}^{19}$ & $\mathrm{JDJ}^{6}$ & $\mathrm{KP}^{20}$ \\
\hline
\end{tabular}

\section{A. Turbulence transport}

One of the motivations of higher-order RANS is the ability to explicitly account for the turbulence transport term by solving an evolution equation of a higher-order moment (equations (2) and (3)). In contrast to $\mathrm{SMC}$, for which the triple-velocity correlation terms have been approximated by various approaches - with limited success ${ }^{2}$ - for third- and fourth-order transport schemes, the task of modeling turbulence transport is delegated to approximating the fourth- and fifth-order moments. The models for $\mathcal{T}$ listed in Table 1 can be classified as PDF approximations, gradient-diffusion models, ${ }^{21}$ scale-similarity models (universal profiles in proportion to a relevant time scale), or a combination of these. The PDF and scale-similarity models are local models, as they relate the higher-order to lower-order moments. Unlike in second-moment equations, the turbulence transport term plays a dominant role in third-moment transport equations. In the near-wall region its contribution is of leading order (figures 5-6). The transport terms involve gradients of fourth-order moments. Hence, high-fidelity fourth-order moment models are essential to predicting the evolution of triple correlations accurately.

The widely used fourth-order moment model by Millionshtchikov, ${ }^{5}$ which makes use of a quasi-normal (QN) hypothesis, assumes the velocity fluctuations have a Gaussian distribution. It relates the fourth-order moment to second-order moments:

$$
\text { M: } \quad \overline{u_{i} u_{j} u_{l} u_{m}}=\left.\overline{u_{i} u_{j} u_{l} u_{m}}\right|_{Q N} \equiv \overline{u_{i} u_{j}} \cdot \overline{u_{l} u_{m}}+\overline{u_{j} u_{l}} \cdot \overline{u_{i} u_{m}}+\overline{u_{l} u_{i}} \cdot \overline{u_{m} u_{j}}
$$

(The label at the start of the above, and following, equations corresponds to the entry in Table 1.) Equation (11) is independent of skewness, as odd correlations are zero for a Gaussian variable. Implicit in this model is the assumption that the flatness is three.

Corrections to $\mathrm{M}$ have been proposed by a number of researchers, including modeling the cumulants (measures of the deviation from Gaussianity $\overline{u^{4}}-\left.\overline{u^{4}}\right|_{Q N}$ ) as a transport process. An algebraic model for the 
cumulant transport by Kawamura et al. ${ }^{16}$ approximates the cumulant as a gradient-diffusion process:

$$
\text { KSK1: } \quad \overline{u_{i} u_{j} u_{l} u_{k}}-\left.\overline{u_{i} u_{j} u_{l} u_{k}}\right|_{Q N}=C_{T} \overline{u_{k} u_{m}} \tau \frac{\partial}{\partial x_{m}} \overline{u_{i} u_{j} u_{l}},
$$

where $C_{T}=0.02$ has been evaluated using the unstrained channel DNS. The turbulence time scale $(\tau)$ is defined as $\tau \equiv k / \varepsilon$. Unlike the even-order moments, triple correlations have inflectional profiles (figures 5 , $6)$. Hence their gradients greatly influence the cumulants.

The non-local model of Cheng et al. ${ }^{17}$ for wall-normal fluctuation accounts for the distribution of triple correlations throughout the domain:

$$
\mathrm{CCH}: \quad \frac{\partial}{\partial y}\left(\overline{v^{4}}-\left.\overline{v^{4}}\right|_{Q N}\right)=C_{1} \frac{\overline{v^{3}}}{\tau} \Longrightarrow \overline{v^{4}}=\left.\overline{v^{4}}\right|_{Q N}+C_{1} \int_{0}^{1} \frac{\overline{v^{3}}}{\tau} d y,
$$

where $C_{1}=4$ and $y=y_{w} / \delta$. Triple correlations are much smaller in magnitude than fourth-order correlations, and are not positive-definite. Hence corrections through this model are expected to be insignificant. This model is easy to implement because the turbulence transport term is the gradient of the fourth-order correlation.

The local model by Gryanik et al. ${ }^{18}$ accounts for the non-Gaussian nature of the flow by making the fourth-order moment a function of the skewness. The model is developed as a solution to the 16-delta PDF function model ${ }^{18}$ that satisfies additional constraints of tensor invariance, symmetry and realizability of the flow variables. The modeled moment is $\overline{u_{i} u_{j} u_{l} u_{m}}=\left.a \overline{u_{i} u_{j} u_{l} u_{m}}\right|_{Q N}$, where the coefficient $a$ is unique for each component, given as

$$
\text { GHRS: } \quad a_{\overline{u^{4}}}=1+\frac{1}{3} S_{1}^{2} ; a_{\overline{v^{4}}}=1+\frac{1}{3} S_{2}^{2} ; a_{\overline{u^{2} v^{2}}}=1+\frac{C_{\overline{u v}}}{1+C \overline{u v}} S_{1} S_{2},
$$

where $S_{\alpha}$ is the skewness of $u_{\alpha}, C_{\overline{u v}}=\overline{\hat{u} \hat{v}}$, and the circumflex ' ' ' denotes normalization by the respective root-mean-square (RMS) value. The cumulant transport here is linear in $\left.\overline{u_{i} u_{j} u_{l} u_{m}}\right|_{Q N}$, leading to a large correction in regions where the product of QN flatness and skewness is high. Also, the correction is only positive for autocorrelations as it involves the square of skewness.

Grossman and Narayan $(1993)^{19}$ proposed a model for the wall-normal component, similar to GHRS, with

$$
\mathrm{GN}: \quad a_{\overline{v^{4}}}=0.767+0.6 S_{2}^{2} .
$$

This model produces flatness $F_{2}<3$, contrary to what is observed from boundary-layer DNS $^{6}$ as well as our current channel DNS (figure 4).

Realizability constraints for third-order correlations can be found from the Cauchy-Schwarz inequality, which states that $\left(\overline{u_{i} u_{j}}\right)^{2} \leq \overline{u_{i}^{2}} \cdot \overline{u_{j}^{2}}$, and

$$
\left(\overline{u_{i} u_{j} u_{k}}\right)^{2} \leq \min \left\{\begin{array}{l}
\overline{u_{i}^{2}}\left(\overline{u_{j}^{2} u_{k}^{2}}-\overline{u_{j}^{2}} \cdot \overline{u_{k}^{2}}\right), \\
\overline{u_{j}^{2}}\left(\overline{u_{i}^{2} u_{k}^{2}}-\overline{u_{i}^{2}} \cdot \overline{u_{k}^{2}}\right), \\
\overline{u_{k}^{2}}\left(\overline{u_{i}^{2} u_{j}^{2}}-\overline{u_{i}^{2}} \cdot \overline{u_{j}^{2}}\right) .
\end{array}\right.
$$

This constraint relates the flatness $(F)$ to the skewness $(S)$, giving $F \geq 1+S^{2}$. It can also be used to relate the third- and second-order moment by invoking the QN hypothesis. Realizability limiters can be designed for algebraic or differential-transport models of higher-order moments.

The turbulent transport terms in the fourth-moment transport equations have a less significant role than they do in the third-order moment transport (cf. figures 5 and 6 with 7 and 8 ). The transport terms involve gradients of fifth-order moments. Models for the fifth-order and higher moments have been proposed by Jovanović et al., ${ }^{6}$ in terms of a Gram-Charlier series-expansion of various orders for the probability density distributions of the turbulence fluctuations about a Gaussian/normal PDF (with the accuracy presumably increasing with increasing order of truncation). The fourth-order truncation is given by 


$$
\begin{aligned}
\overline{u^{4} v} & =6 \overline{u^{2} v} \cdot \overline{u^{2}}+4 \overline{u v} \cdot \overline{u^{3}}, \\
\overline{u^{3} v^{2}} & =6 \overline{u v} \cdot \overline{u^{2} v}+3 \overline{u^{2}} \cdot \overline{u v^{2}}+\overline{v^{2}} \cdot \overline{u^{3}}, \\
\overline{u^{2} v^{3}} & =6 \overline{u v} \cdot \overline{u v^{2}}+3 \overline{v^{2}} \cdot \overline{u^{2} v}+\overline{u^{2}} \cdot \overline{v^{3}}, \\
\overline{u v^{4}} & =6 \overline{u v^{2}} \cdot \overline{v^{2}}+4 \overline{u v} \cdot \overline{v^{3}}, \\
\overline{v^{5}} & =10 \overline{v^{3}} \cdot \overline{v^{2}} \\
\overline{u^{5}} & =10 \overline{u^{3}} \cdot \overline{u^{2}} .
\end{aligned}
$$

The fifth-order moments from the DNS are inflectional. ${ }^{8}$ Note that equation (17) assumes the inflectional points of the fifth-order moments to be same as those of the third-order moments. This assumption agrees reasonably well with DNS, as we notice second-order turbulence transport term (figure 9 in Ref. 7) to have a similar trend as fourth-order turbulence transport term (figure 7). The non-dimensionalized values of fifthorder moments are represented as super-skewness, $\mathrm{SS} \equiv \overline{\hat{u}}^{5}$. The magnitude of the fifth-order skewness is larger than the third-order skewness $(|\mathrm{SS}|>|\mathrm{S}|)$ for all velocity components. All the terms in equation (17) have correct near-wall asymptotic behavior as $y_{w} \rightarrow 0$. The JDJ model has been shown to provide a good approximation for the higher-order moments in a channel flow ${ }^{6}$ and in a longitudinally rotating pipe. ${ }^{20}$

Nagano and Tagawa ${ }^{22}$ have developed a 'structural' model for the triple correlations based on the sweep and ejection mechanisms (i.e. the so-called Q4 and Q2 events, respectively) in near-wall turbulence. The model relates the third-order 'shear' stresses to the skewness as

$$
\overline{\hat{u}^{m} \hat{v}^{n}}=C\left[\sigma_{m} \overline{\hat{u}^{3}}+\sigma_{n} \overline{\hat{v}}^{3}\right],
$$

where $(n, m)=(1,2)$ or $(2,1)$ and

$$
C=\frac{1}{3\left[\left(\frac{\pi}{2}\right)^{2}-1\right]}, \text { with } \sigma_{x}=\left\{\begin{array}{r}
-\frac{\pi}{2}, x \text { is even } \\
1, x \text { is odd }
\end{array}\right.
$$

Hence, while modeling third-order moments, only transport equations for $\overline{u^{3}}$ and $\overline{v^{3}}$ need to be solved and triple cross-correlations such as $\overline{u v^{2}}$ and $\overline{u^{2} v}$ can be predicted from equation (18).

Based on this idea, here we define a new model for turbulence transport terms in the fourth-order moment equations. Since the fifth-order correlations are structurally similar to the third-order correlations (as they measure the imbalance between Q4 and Q2 contributions), Nagano and Tagawa's structural model (18) can be extended to write the fifth-order cross-component moments in terms of the super-skewness as

$$
\text { JCR1: } \quad \overline{\hat{u}^{m} \hat{v}^{n}}=C\left[\sigma_{m} \overline{\hat{u}^{5}}+\sigma_{n} \overline{\hat{v}^{5}}\right],
$$

where $m+n=5$, and the model coefficients $C, \sigma_{m}$ and $\sigma_{n}$ are the same as in equation (18). This new model can be used to predict the cross-correlations using autocorrelations, by either solving for transport equations for $\overline{u^{5}}$ and $\overline{v^{5}}$ or by using an algebraic model that relates super-skewness to skewness. We will include this new JCR1 model in the comparisons in Section VI.

\section{B. Velocity-pressure-gradient correlation}

As mentioned earlier, a few modelers extend the splitting of velocity-pressure-gradient (into pressure-strain plus pressure transport) to higher-order moments. Pressure-strain is modeled by Dekeyser and Launder ${ }^{3}$ by the same strategy typically used in SMC. There is a linear return-to-isotropy term ('slow' part) and a set of production terms ('fast' part):

$$
\text { DL1: } \quad \phi_{i j l}=-C_{1} \frac{\overline{u_{i} u_{j} u_{l}}}{\tau}-C_{2}\left(\mathcal{P}_{i j l}^{S}+\mathcal{P}_{i j l}^{T}\right),
$$

with constants $C_{1}=13.3 \overline{3}$ and $C_{2}=0.5$. Nagano and Tagawa ${ }^{14}$ proposed another model for pressure-strain,

$$
\mathrm{NT}: \quad \phi_{i j l}=\frac{-1}{C_{u 1}} \frac{\overline{u_{i} u_{j} u_{l}}}{\tau}+C_{u 2} \tau \overline{u_{i} u_{m}} \frac{\partial}{\partial x_{m}}\left[\frac{1}{\tau}\left(\overline{u_{i}^{2}}-\frac{2}{3} k\right)\right],
$$


with the model constants $C_{u 1}=0.12$ and $C_{u 2}=1.4$. The second part in this model is not directly dependent on strain (there is no 'fast' part). Hence response to changes in mean strain are indirectly resolved through changes in Reynolds stress, potentially lagged. Moreover, the second part is identical for the $\phi_{111}, \phi_{112}$, and $\phi_{122}$ components, indicating these components have similar dynamics. The model of Kurbatskii and Poroseva ${ }^{4}$ for the velocity-pressure-gradient of the third-order moment is given by

$$
\mathrm{KP} 1: \quad \Pi_{i j l}=b_{i j l}+a_{m i j l n} \partial_{n} U_{m} .
$$

This model consists of a 'slow' part $\left(b_{i j l}\right)$ and a strain-dependent 'fast' term. The 'slow' part is a function of three coefficients and the tensor $a_{m i j l n}$ is dependent on two model coefficients. This model will not be tested as the full expression of the model is not available in the open literature.

An improvement to the model of Dekeyser and Launder ${ }^{3}$ was proposed by Kawamura et al., ${ }^{16}$ in terms of the velocity-pressure-gradient:

$$
\text { KSK2: } \quad \Pi_{i j l}=\Pi_{i j l}^{*}\left(1-f_{w 2}\right)+\phi_{i j l} f_{w 2},
$$

where

$$
\begin{aligned}
\Pi_{i j l}^{*} & =-\frac{1}{\tau}\left(\overline{u_{i} u_{j} u_{m}} n_{m} n_{l}+\overline{u_{j} u_{l} u_{m}} n_{m} n_{i}+\overline{u_{l} u_{i} u_{m}} n_{m} n_{j}\right), \\
\phi_{i j l} & =-C_{1} \frac{1}{\tau}\left\{3 \overline{u_{i} u_{j} u_{l}}-C_{1}^{\prime}\left(\overline{u_{m} u_{m} u_{i}} \delta_{j l}+\overline{u_{m} u_{m} u_{j}} \delta_{l i}+\overline{u_{m} u_{m} u_{l}} \delta_{i j}\right)\right\}-C_{2}\left(\mathcal{P}_{i j l}^{S}+\mathcal{P}_{i j l}^{T}\right),
\end{aligned}
$$

with empirical constants $C_{1}=0.3, C_{1}^{\prime}=0.6$ and $C_{2}=0.1$. In equation (23), a modified $\phi_{i j l}$ was provided (compare with equation (20)), and a correct wall-asymptotic behavior was included by damping components as a function $\left(f_{w 2}\right)$ of $\overline{v^{3}}$. The model assumes $\Pi_{i j l}=\phi_{i j l}$ away from the wall and $\Pi_{i j l}$ is only dependent on a return-to-isotropy term in the near-wall region.

An approach to modeling the velocity-pressure-gradient correlation is proposed here. While not a model per se (because it relies on an additional unspecified model for $\mathcal{T}_{i j l}$ ), this approach will be tested in an $a$ priori sense in Section VI, with $\mathcal{T}_{i j l}$ taken from the DNS. The approach is derived from equation (2), and models the $\Pi_{i j l}$ term as

$$
\text { JCR2: } \quad \Pi_{i j l}=C_{a} \frac{\overline{u_{i} u_{j} u_{l}}}{\tau}-C_{b}\left(\mathcal{P}_{i j l}^{S}+\mathcal{P}_{i j l}^{T}+\mathcal{T}_{i j l}\right) .
$$

The first term on the right-hand side represents the 'slow' term, where $C_{a} / \tau$ accounts for the turbulence response, and the remaining terms account for the 'fast' part. The 'fast' part only has contributions from production due to shear and turbulence and turbulence transport, as they are dominant (figures 5 and 6). While dissipation has a role in the budget transport, its model expression (discussed in the next section) is not well known. Therefore, its effects are resolved instead through tuning the coefficients, chosen as $C_{a}=0.5$ and $C_{b}=0.9$.

Currently, there exists no model for the fourth-order velocity-pressure-gradient tensor. We propose an approach similar to the description in equation (24), with

$$
\text { JCR3,4: } \quad \Pi_{i j l m}=C_{a} \frac{\overline{u_{i} u_{j} u_{l} u_{m}}}{\tau}-C_{b}\left(\mathcal{P}_{i j l m}^{S}+\mathcal{P}_{i j l m}^{T}+\mathcal{T}_{i j l m}\right) .
$$

As with JCR2, this is not a complete model because $\mathcal{T}_{i j l m}$ is currently taken from the DNS. The effects of diffusion are again accounted for by the coefficient $C_{a}$. This choice is based on the observation that dissipation is self-similar to the moment it transports (refer to the next section). This approach was tested for fourthorder correlations, and we find that including the 'fast' part improves the predictions for wall-normal auto and cross-correlations. The simpler approach, JCR3, only accounts for the 'slow' part in equation (25) by using $C_{a}=2, C_{b}=0$, that is, assuming similarity with the fourth-order moments. The JCR4 version accounts for both the 'slow' and strain-dependent 'fast' part with $C_{a}=1.5, C_{b}=0.3$. Note that $C_{a}<C_{b}$ in equation (24), while $C_{a}>C_{b}$ in equation (25), indicating that $\Pi_{i j l m}$ is more scale-similar to the moment it transports than is $\Pi_{i j l}$.

\section{Dissipation}

The majority of the models for dissipation in SMC assume isotropy and apply a near-wall correction to approach the wall values. One advantage to modeling of high-order dissipation is that the wall values of 
dissipation for moments greater than second-order are zero. This leads to a simple boundary condition for dissipation. However, the near-wall asymptote as $y_{w} \rightarrow 0$ needs to be modeled through damping or other means. Assuming local isotropy of third-order moment dissipation leads to $\varepsilon_{i j l}=0$; such a model was used by André et al. ${ }^{1}$ (A). From the DNS, we notice that such a model is grossly incorrect, so it will not be included in the testing below.

A more accurate model, by Dekeyser and Launder, ${ }^{3}$ relates $\varepsilon_{i j l}$ to its lower-order term by invoking a generalized gradient-diffusion hypothesis ${ }^{21}(\mathrm{GGDH})$. They further assume isotropy of second-order moment dissipation, that is $\varepsilon_{i j}=(2 / 3) \varepsilon \delta_{i j}$, with $\varepsilon \equiv\left|\varepsilon_{l l}\right|$ to relate it to the scalar diffusion. The model is

$$
\text { DL2: } \quad \varepsilon_{i j l}=C_{\varepsilon} \tau \frac{\partial \varepsilon}{\partial x_{k}}\left(\overline{u_{l} u_{k}} \delta_{i j}+\overline{u_{j} u_{k}} \delta_{l i}+\overline{u_{i} u_{k}} \delta_{j l}\right),
$$

with $C_{\varepsilon}=1$. However, equation (26) results in $\varepsilon_{111}=3 \varepsilon_{122}$ and $\varepsilon_{222}=3 \varepsilon_{112}$, whereas the channel DNS shows these values to be unequal in magnitude and sign. Note that third-order moment 'dissipation' is not dissipative, as the components are not monotonically negative in the channel flow. ${ }^{8}$ The inflectional profile of $\varepsilon_{i j l}$ will not be reproduced by this model because the gradient of isotropic dissipation is always non-negative in a channel flow. This shortcoming can be overcome by reverting to the GGDH model, without assumptions of isotropy on the second-order dissipation. Such an anisotropic model of DL2 (with $C_{\varepsilon}=1$ ) is

$$
\text { DL3: } \quad \varepsilon_{i j l}=C_{\varepsilon} \tau\left(\overline{u_{l} u_{k}} \frac{\partial \varepsilon_{i j}}{\partial x_{l}}+\overline{u_{j} u_{l}} \frac{\partial \varepsilon_{i k}}{\partial x_{l}}+\overline{u_{i} u_{l}} \frac{\partial \varepsilon_{j k}}{\partial x_{l}}\right) .
$$

A model based on similarity of $\varepsilon_{i j l}$ to the third-order moments was developed by Kawamura et al. ${ }^{16}$ The expected near-wall damping (refer to the Appendix) is provided using the invariants of the Reynolds stress anisotropy and the componential damping using a wall-normal vector $\left(n_{i}\right)$. The model (with constant $C_{\varepsilon}=2$ ) is

$$
\mathrm{KSK} 3: \quad \varepsilon_{i j l}=C_{\varepsilon} \frac{\overline{u_{i} u_{j} u_{k}}}{\tau} f_{w 1}+\varepsilon_{i j l}^{*}\left(1-f_{w 1}\right) \text {, }
$$

where,

$$
\begin{aligned}
\varepsilon_{i j l}^{*} & =\frac{1}{\tau}\left\{3 \overline{u_{i} u_{j} u_{l}}+2\left(\overline{u_{i} u_{j} u_{m}} n_{m} n_{l}+\overline{u_{j} u_{l} u_{m}} n_{m} n_{i}+\overline{u_{l} u_{i} u_{m}} n_{m} n_{j}\right)\right. \\
& \left.+\left(\overline{u_{i} u_{m} u_{k}} n_{m} n_{k} n_{j} n_{l}+\overline{u_{j} u_{m} u_{k}} n_{m} n_{k} n_{l} n_{i}+\overline{u_{l} u_{m} u_{k}} n_{m} n_{k} n_{i} n_{j}\right)\right\} .
\end{aligned}
$$

The GGDH has been extended to the fourth-order dissipation tensor by Kurbatskii and Poroseva. ${ }^{20}$ The resulting expression for dissipation is

$$
\begin{aligned}
\mathrm{KP} 2: \quad \varepsilon_{i j l m}=-C_{E 4} \frac{k}{\varepsilon} & \left(\overline{u_{i} u_{j} u_{k}} \frac{\partial \varepsilon_{l m}}{\partial x_{k}}+\overline{u_{i} u_{l} u_{k}} \frac{\partial \varepsilon_{j m}}{\partial x_{k}}+\overline{u_{i} u_{m} u_{k}} \frac{\partial \varepsilon_{j l}}{\partial x_{k}}\right. \\
& \left.+\overline{u_{j} u_{l} u_{k}} \frac{\partial \varepsilon_{i m}}{\partial x_{k}}+\overline{u_{j} u_{m} u_{k}} \frac{\partial \varepsilon_{i l}}{\partial x_{k}}+\overline{u_{l} u_{m} u_{k}} \frac{\partial \varepsilon_{i j}}{\partial x_{k}}\right) .
\end{aligned}
$$

We find $C_{E 4}$ to not be a constant for the components of dissipation; however, a value of 2 gave a reasonable fit to the DNS (refer to following section).

In the fourth-order budgets, the dissipation term $\varepsilon_{i j l m}$ is always dissipative (figures 7 and 8 ). We propose a new model based on similarity of dissipation to the fourth-order moment. One of the weaknesses of such a model is that free-stream values of dissipation are incorrect, as at the channel centerline $\overline{u_{i} u_{j} u_{l} u_{m}}$ are non-zero while dissipation $\varepsilon_{i j l m}$ is zero. Two variants are proposed, where the fourth-order timescale is $\tau_{4} \equiv \overline{u_{i} u_{j} u_{i} u_{j}} / \varepsilon_{l m l m}$ and $\tau_{2}$ is based on the second-order timescale $\left(\tau_{2} \equiv \overline{u_{i} u_{i}} / \varepsilon_{j j}\right)$. The constants $C_{1}=2=2 C_{2}$ are recommended:

$$
\begin{aligned}
& \text { JCR5 : } \quad \varepsilon_{i j l m}=C_{1} \frac{\overline{u_{i} u_{j} u_{l} u_{m}}}{\tau_{2}}, \\
& \text { JCR6 : } \quad \varepsilon_{i j l m}=C_{2} \frac{\overline{u_{i} u_{j} u_{l} u_{m}}}{\tau_{4}} .
\end{aligned}
$$




\section{A priori model testing}

In this section, closure-model components of all $u$ and $v$ correlations of third- and fourth-order moments are tested a priori - that is, the individual closure assumptions are compared to the corresponding DNS results by using component DNS data as input to the models. The limitations of this approach are acknowledged, namely that a RANS scheme's overall behavior is due to the combined effect of all the closure assumptions, which in general will include many compensating errors, such that a scheme's net prediction may be much better in practice than implied by the validity of the individual assumptions. A complete picture will require a posteriori tests of an entire scheme (as in Sciberras and Coleman ${ }^{10}$ ). This limitation notwithstanding, a model developer will presumably benefit by knowing the accuracy of the individual closure assumptions, which motivates the present study. The main goals are to assess: (i) the ability of the individual closure assumptions to reproduce the statistics of the DNS, (ii) the effectiveness of model coefficients, tuned for an optimal fit of all components, ${ }^{16}$ and (iii) the merits of general modeling concepts, and thereby their potential usefulness for 2D attached and APG flows.

\section{A. Unstrained channel}

The unstrained channel flow is a steady fully developed channel flow. That is, the moments of the statistics do not evolve with time, as the budget terms balance each other, leading to $\partial_{t}=0$ in equations (2) and (3). The flow is in turbulence 'equilibrium', with the production of turbulence kinetic energy roughly balancing dissipation in the (nominal) log layer of the flow. ${ }^{10}$ We also notice 'equilibrium' in the fourth-order turbulence kinetic energy analog (figure 11). The statistics presented here are representative of a moderate Reynolds number $\left(R e_{\tau}=392\right)$ channel flow, which is about three times larger than that required to sustain a turbulent flow. ${ }^{10}$

For closure at the third-moment level, turbulence transport models are important. From equation (3), we notice that the fourth-order moments are functions of the third-order moment through the production due to turbulence term $\left(\mathcal{P}_{i j l}^{T}\right)$. There is no dependence on the Reynolds stresses, except through gradients in the $\mathcal{P}_{i j l}^{T}$ term. However, the most commonly used turbulence transport models for third-order moments are based on the Gaussian PDF approximation ${ }^{5}(\mathrm{M})$ of fluctuating velocities. This model expresses the fourthorder moments as products of second-order moments. Figure 12 shows model comparisons with the DNS data. Note that in this particular case only the 122 and 222 components enter the solution, but we include the 111 and 112 components for completeness. The basic M model tends to over-predict the magnitude of the peak value for $\mathcal{T}_{111}$ and $\mathcal{T}_{112}$, and the peak is predicted to be too far from the wall for $\mathcal{T}_{222}$. This offset could be critical, as $\overline{v^{3}}$ will be overpredicted near the wall, indirectly affecting the wall shear stress, an important quantity in APG flows. The other models, representing corrections to the M model, do not improve the comparisons at all. In some cases the corrected model has no noticeable impact, while in others the corrected model is worse. For example, the GN model yields too small a peak magnitude for the 222 component and the KSK1 model yields too large a peak magnitude for all components.

Fourth-order turbulence transport terms may serve as either a source or sink (figures 7,8 ). The JDJ model predicts the profiles reasonably well as seen in figure 13, with some errors near the local minima/maxima. The nearest-wall peaks in $\mathcal{T}_{2222}$ and $\mathcal{T}_{1222}$ are offset away from the wall. The magnitudes of the second (larger) peaks are overpredicted for all components except $\mathcal{T}_{2222}$. The structural turbulence model (JCR1) presented in equation (19) for the cross-correlations uses the JDJ model to predict autocorrelations $\overline{u^{5}}$ and $\overline{v^{5}}$. Predictions using this model are comparable to those of JDJ, with an improved prediction of the 1111 component and under-prediction of 1222 component. Note that JCR1 does not provide a prediction for $\mathcal{T}_{2222}$.

Third-order velocity-pressure-gradient terms are sources near the wall $\left(y_{w} / \delta<0.2\right)$, contributing to the production of $\overline{v^{4}}$ and $-\overline{u v^{3}}$ (figures 5,6 ). Further away from the wall they are a mild sink. These are dominant terms in the buffer region. The models NT, KSK2, DL1, and JCR2 include the net effect of a linear term ('slow' response) which is a function of the third-order moment and a non-linear term ('fast' response) which is a function of third-order moment, strain, Reynolds stresses and its gradients. Comparisons are plotted in figure 14. Generally, all models are poor, indicating that this component of turbulence modeling is in need of new ideas with improved capabilities. Recall that both NT and DL1 model the pressure-strain term, rather than velocity-pressure-gradient. Therefore both the $\Pi$ and $\phi$ results from the DNS are plotted in figure 14 so that appropriate direct comparisons can be made. It is interesting to note that the DL1 model predicts a profile whose general shape is more representative of $\Pi_{222}$ than $\phi_{222}$. As the DL1 model is only a 

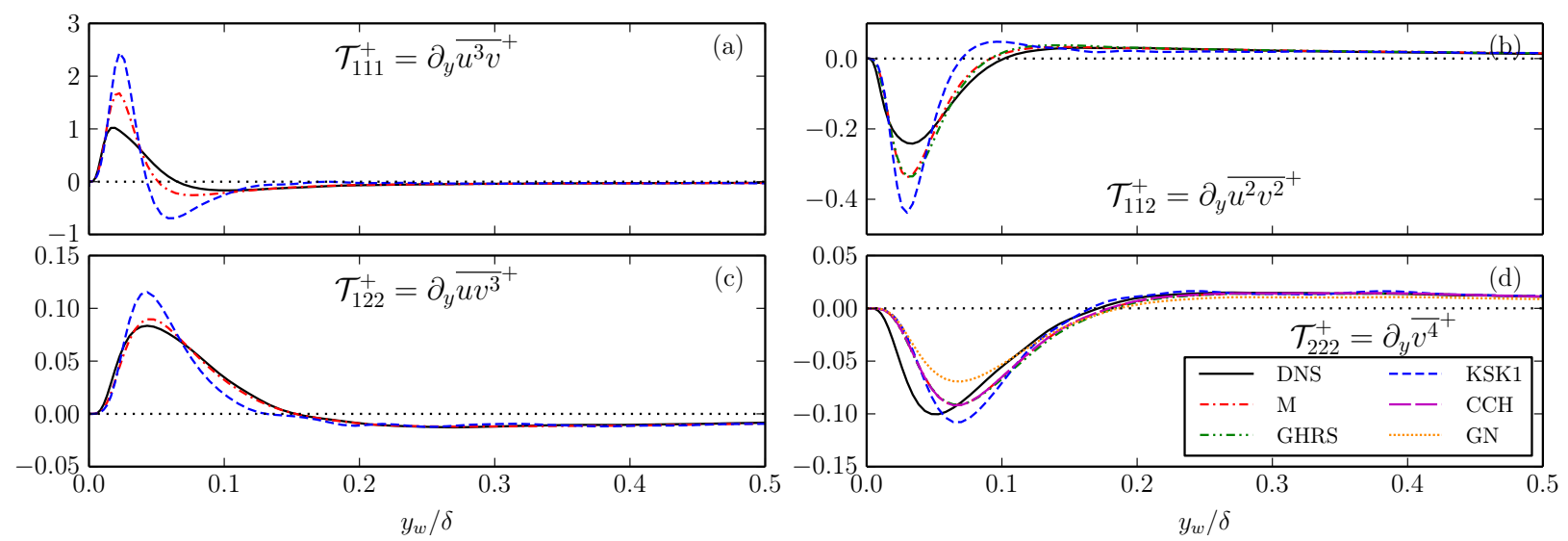

Figure 12. A priori model predictions of third-order turbulence transport term $\left(\mathcal{T}_{i j l}\right)$ in a fully developed channel at $R e_{\tau}=392$. The terms are non-dimensionalized by $u_{\tau}^{4}(0) / \nu$. The reference statistics from DNS are shown as a solid line.
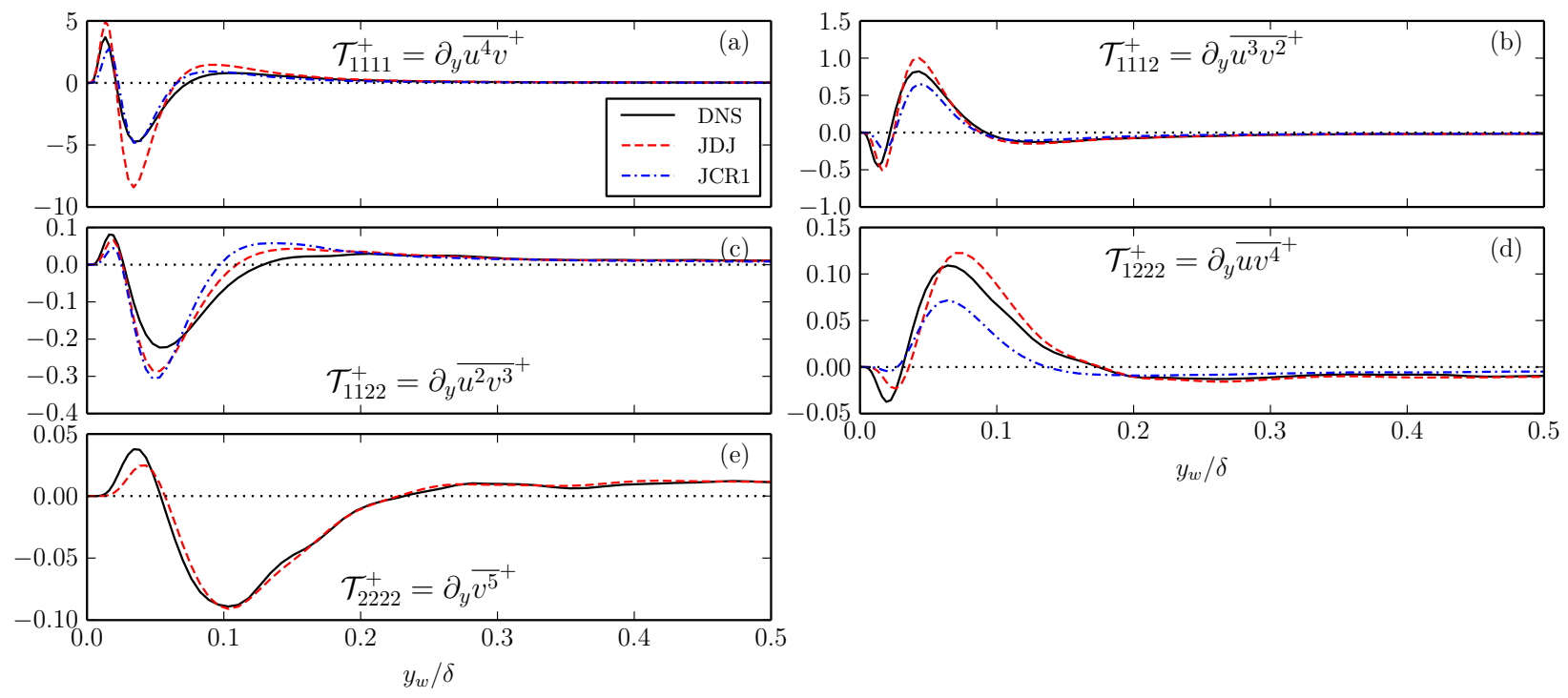

Figure 13. A priori model predictions of fourth-order turbulence transport term $\left(\mathcal{T}_{i j l m}\right)$ in a fully developed channel at $R e_{\tau}=392$. The terms are non-dimensionalized by $u_{\tau}^{5}(0) / \nu$. The reference statistics from DNS are shown as a solid line. 
function of moments and budget terms of the same components, the wall limiting behavior is accurate for all components except $\Pi_{111}$, which is incorrectly predicted to be $\mathcal{O}(y)$. The NT model has a 'slow' part identical to that of DL1 and the model asymptote as $y_{w} \rightarrow 0$ is similar to DL as the 'slow' part is of leading order near the wall. This model has shortcomings similar to the DL1. As mentioned earlier, the strain-dependent term is identical for components 111, 112, and 122 in the NT model. This indicates that the 'slow' term plays an important role distinguishing the components. Both NT and DL1 severely over-predict the peaks and valleys for $\phi_{111}$.

Among the models for $\Pi$, the KSK2 model is a blended model (equation (23)), considering a 'slow' part only in the near wall region. This part is sensitized to the wall-normal vector so as to predict the correct asymptotic damping as $y_{w} \rightarrow 0$. The JCR2 model has a 'fast' term that accounts for turbulent transport $\left(\mathcal{T}_{i j l}\right)$ in addition to the production term. This model has a 'slow' part similar to the DL1 model and hence has the same near-wall shortcoming for the $\Pi_{111}$ component. The JCR2 approach yields good results for all but the 111 component, but recall that this is strictly not a model because DNS values for $\mathcal{T}_{i j l}$ are used in its definition. The results from JCR2 indicate that if an accurate model for $\mathcal{T}_{i j l}$ were included, then it would yield the results shown here for $\Pi_{i j l}$.
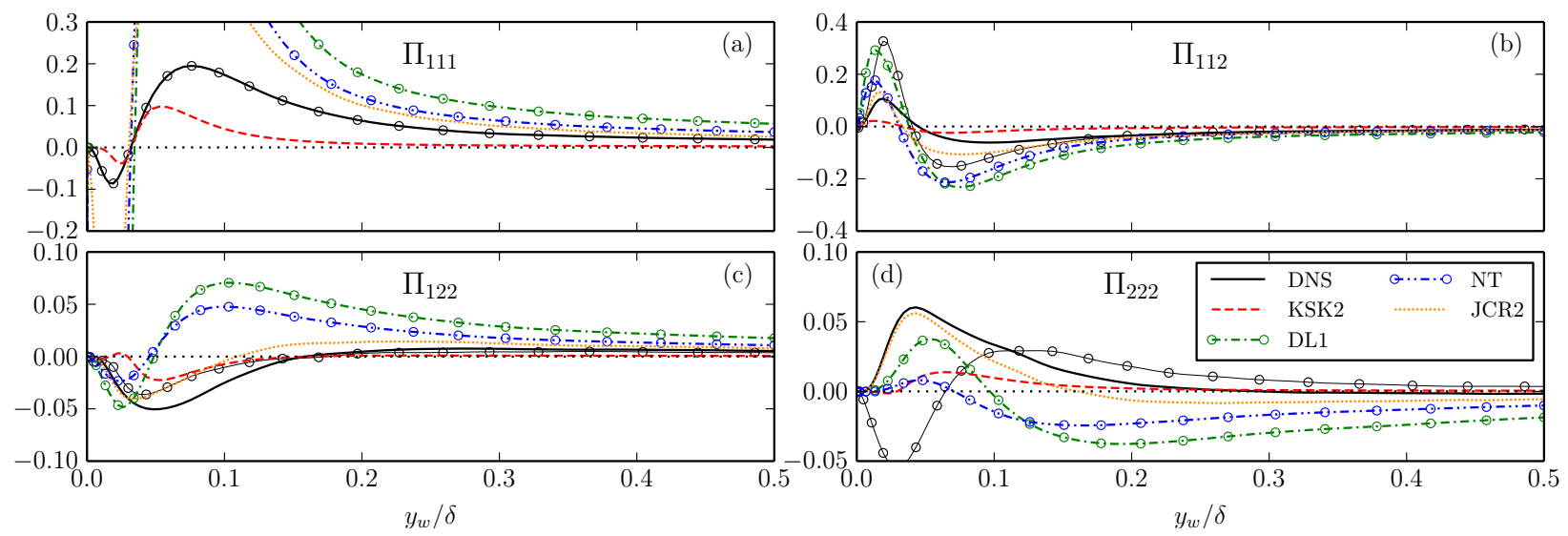

Figure 14. A priori model predictions of third-order velocity-pressure-gradient budget term $\left(\Pi_{i j l}\right)$ in a fully developed channel at $R e_{\tau}=392$. The terms are non-dimensionalized by $u_{\tau}^{4}(0) / \nu$. The reference $\Pi_{i j l}$ from DNS is shown as —, with pressure-strain term $\phi_{i j l}$ as $\_$-
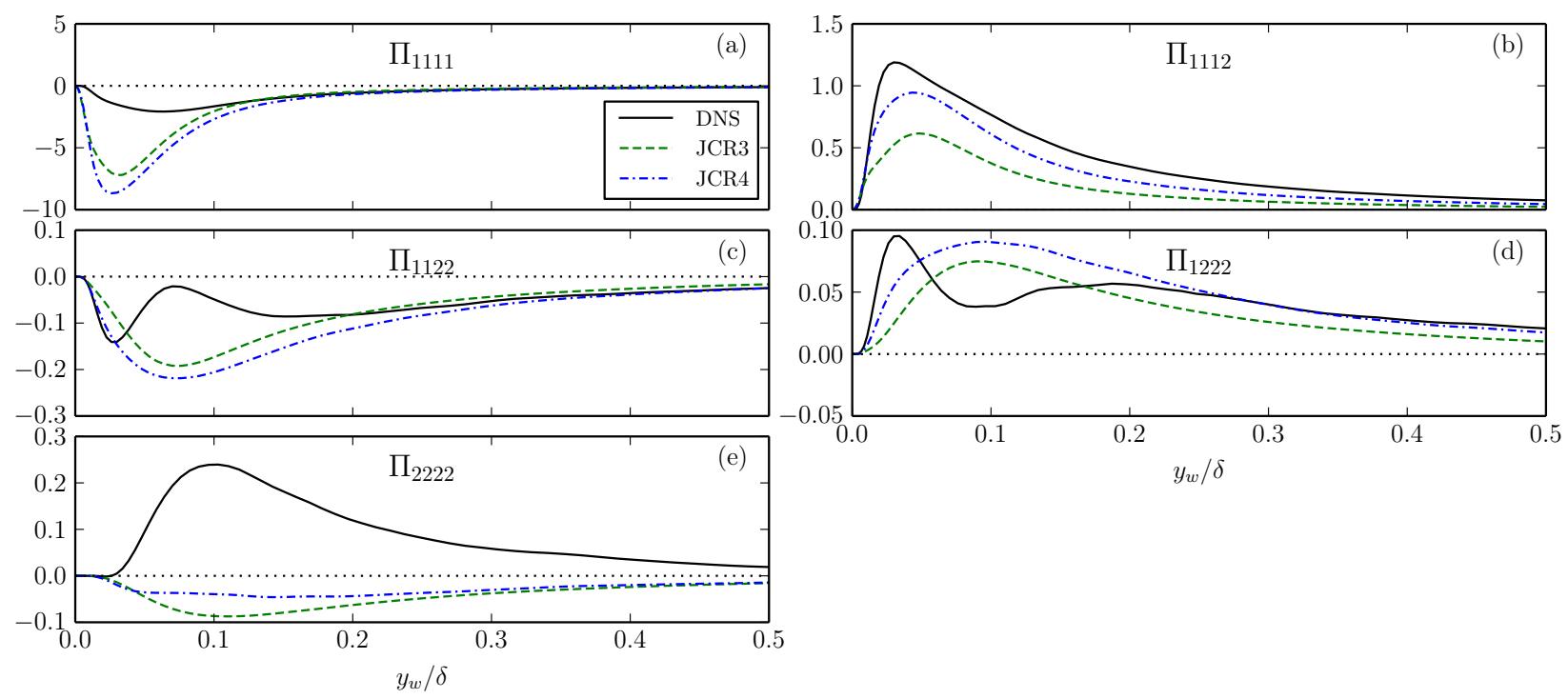

Figure 15. A priori model predictions of fourth-order velocity-pressure-gradient budget term $\left(\Pi_{i j l m}\right)$ in a fully developed channel at $R e_{\tau}=392$. The terms are non-dimensionalized by $u_{\tau}^{5}(0) / \nu$. The reference statistics from DNS are shown as a solid line. 
The fourth-order velocity-pressure-gradient term is very similar to the second-order velocity-pressuregradient term, in that it is one of the dominant terms in the balance. $\Pi_{2222}$ produces $\overline{v^{4}}$ (compare figure 7 to figure 9 in Ref. 7), and $\Pi_{1222}$ produces $\overline{u v^{3}}$ (compare figure 8 to figure 8 in Ref. 7). As shown in figure 15, the JCR3 and JCR4 approaches yield generally poor results compared to DNS, even with exact representations of $\mathcal{T}_{i j l m}$ as a part of their definitions. In particular, they miss the inflectional character of the 1122 and 1222 components, and the sign of the 2222 component is incorrect. Some of the mis-prediction is likely because the dissipation model is not accounted for in the approaches used. Also recall that JCR3 includes only the 'slow' term. So the fact that JCR3 and JCR4 predictions are comparable indicates that the 'fast' term probably does not play a dominant role in $\Pi_{i j l m}$ models. The wall limiting behavior of JCR3 and JCR4 models is accurate for all components except $\Pi_{1111}$, which is incorrectly predicted to reduce at $\mathcal{O}\left(y_{w}^{2}\right)$ as $y_{w} \rightarrow 0$ (refer to the Appendix). Again, as with the third-order velocity-pressure-gradient terms, modeling for the fourth-order velocity-pressure-gradient terms needs improvement.

Third-order dissipation is small relative to other budget terms, acting as a sink in the outer layer, $y / \delta>0.05$ (figures 5,6 ). The DL2 model expresses $\varepsilon_{i j l}$ as a non-linear function of Reynolds stress and gradient of second-order dissipation. As noted earlier, the isotropic dissipation version DL2 cannot predict the inflectional profile of $\varepsilon_{i j l}$. The anisotropic-dissipation model DL3 accounts for the change in sign only for components $\varepsilon_{122}$ and $\varepsilon_{222}$ (figure 16), with the change in sign occurring at a wall distance larger than reference DNS. The DL3 model requires accounting for second-order dissipation anisotropy either through an algebraic or differential model for each of its $\varepsilon_{i j}$ components. The peak magnitudes of dissipation are generally overpredicted by the DL2,3 models, with the largest error in $\varepsilon_{222}$. The KSK3 model considers $\varepsilon_{i j l}$ to be similar to the moment it transports in the outer layer, and near the wall similarity moments are based on wall-normal products as in equation (28). The near-wall behavior of $\varepsilon_{i j l}$ is accurately predicted by KSK3, and it predicts all the dissipation components reasonably well in general.
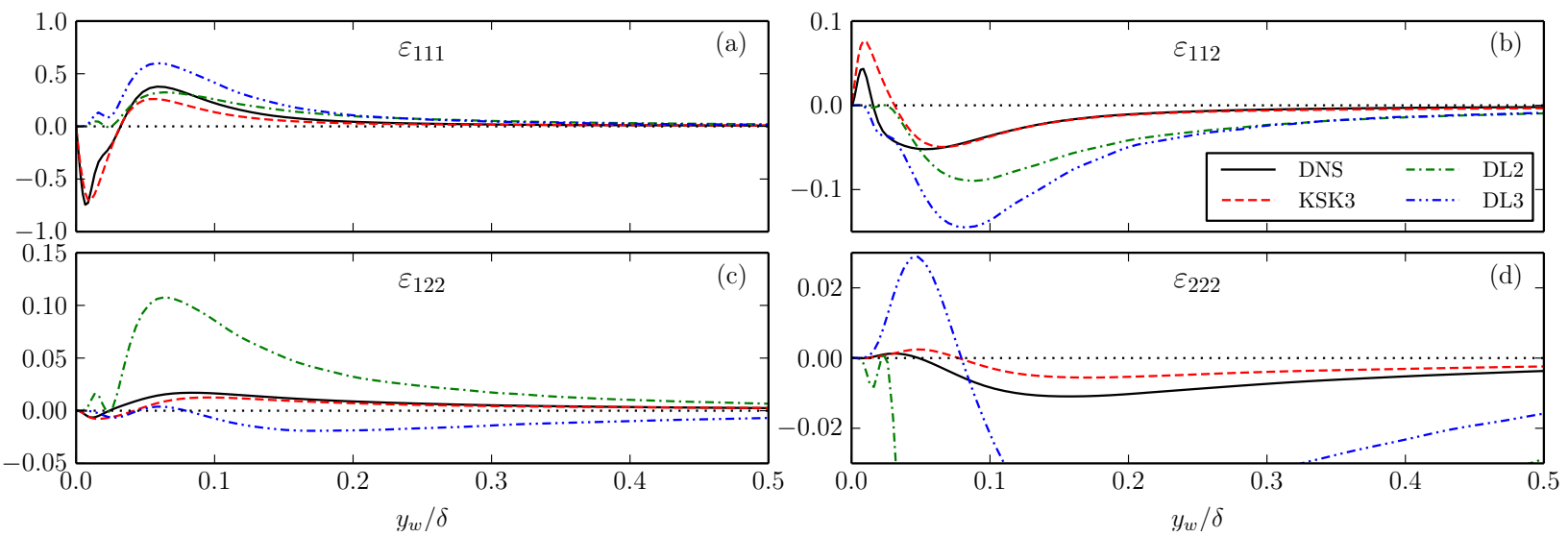

Figure 16. A priori model predictions of third-order dissipation budget term $\left(\varepsilon_{i j l}\right)$ in a fully developed channel at $R e_{\tau}=392$. The terms are non-dimensionalized by $u_{\tau}^{4}(0) / \nu$. The reference statistics from DNS are shown as a solid line.

Fourth-order dissipation plays a dominant 'dissipating' role in the transport of $\overline{v^{4}}$ and $\overline{u v^{3}}$ (figures 7, 8). The GGDH model of KP2 represents $\varepsilon_{i j l m}$ as a non-linear function of third-order moments and second-order dissipation. Due to the dependence on the triple-correlation, the model inherently produces inflectional profiles (figure 17), hence incorrect. The model provides a qualitative comparison to DNS only in the region far away from wall. Although the near-wall asymptotic behaviour of $\varepsilon_{i j l m}$ is accurate, the magnitudes of dissipation are largely underpredicted. The new JCR 5,6 models based on scale similarity consider $\varepsilon_{i j l m}$ to be scale-similar to the corresponding moment it transports. The scaling variable used for JCR5 is the secondorder timescale, while the JCR6 model uses the fourth-order timescale variables defined in Section II. Both the models predict the dissipation components reasonably well, with $\varepsilon_{2222}$ underpredicted in magnitude. The JCR6 model improves the near-wall prediction of dissipation terms, especially $\varepsilon_{1111}$ and $\varepsilon_{1112}$. Both the scale-similarity models predict the correct near-wall asymptotic decay of dissipation. 


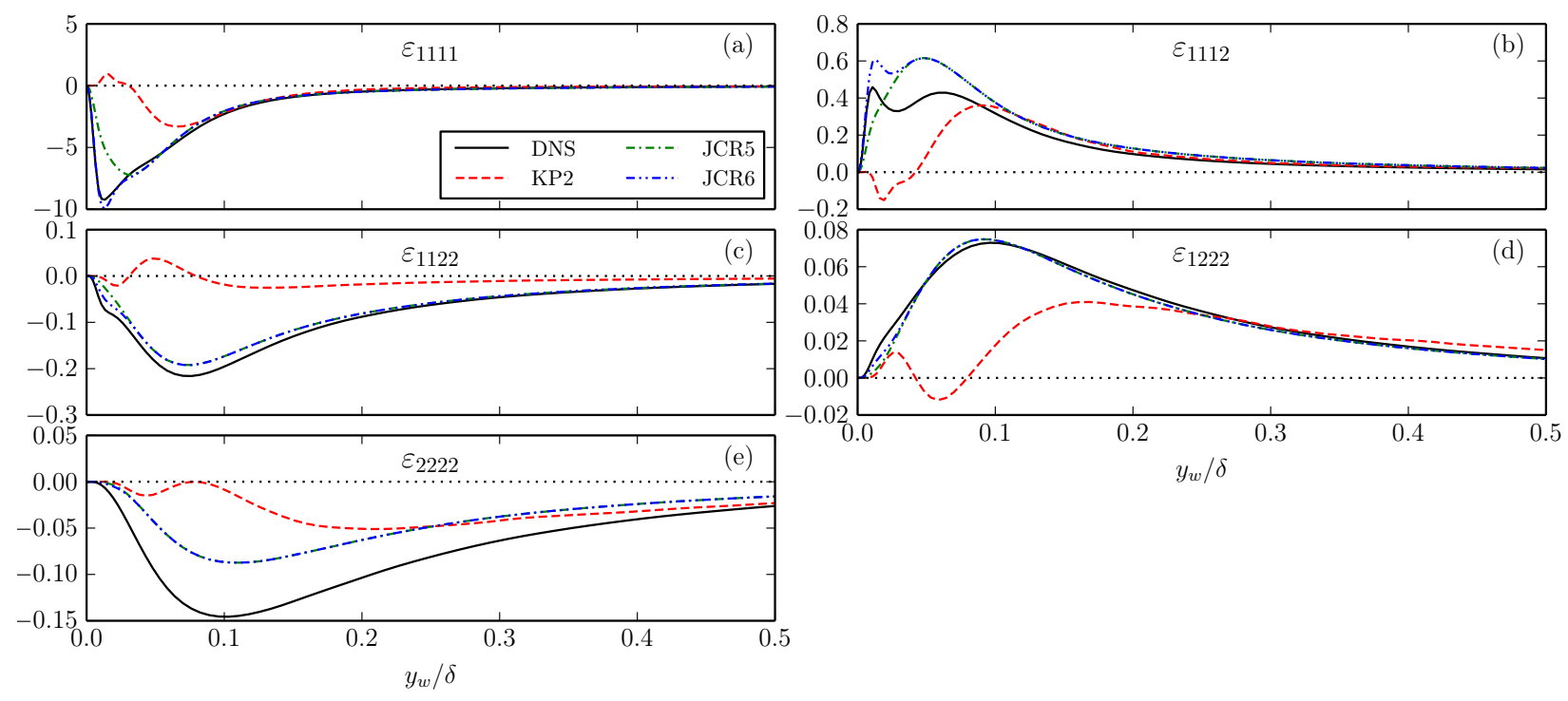

Figure 17. A priori model predictions of fourth-order dissipation budget term $\left(\varepsilon_{i j l m}\right)$ in a fully developed channel at $R e_{\tau}=392$. The terms are non-dimensionalized by $u_{\tau}^{5}(0) / \nu$. The reference statistics from DNS are shown as a solid line. JCR5 and JCR6 model predictions overlap in all regions except near the wall.

\section{B. APG-strained channel}

APG straining of the channel causes the turbulence statistics to reduce in magnitude at different rates in the inner and outer layers ${ }^{7}$ (figures 5-8). From equations (1)-(3), we know that the statistical moments evolve due to the forcing through the production due to applied strain $\left(\mathcal{P}^{A}\right)$. Although the straining does not vary with time, the forcing is time-dependent due to non-linear dependence on moments. The objective of a priori testing of the strained channel is to find out if the models implicitly account for the effects of APG forcing through their dependence on same or lower-order moments. Statistics at a strain time $A_{22} t=0.365$ (sufficiently away from the start of straining so that initial transient effects are negligible) are used to test the models. Similar to the unstrained channel, all $u$ and $v$ correlation components of budget terms are presented. The scales of figures 18-23 are the same as the figures in section A, in order to juxtapose model predictions to the unstrained case and to show reduction in moment budget magnitudes.

Third-order turbulence transport has a reduced magnitude in the near-wall region due to applied strain (figure 18). The 122 and 222 components respond slower to the applied strain than the 111 and 112 components. All the models show similar predictions relative to DNS (figure 18) compared to the unstrained case. The waviness in KSK1 predictions are caused by gradients of third-order moments from DNS, and can be ignored as the gradients magnify minor statistical errors. Overall, the error in modeling predictions has somewhat increased due to straining.

For the fourth-order $\mathcal{T}_{i j l m}$ statistics (figure 19), the JDJ model predicts the reduction in magnitude of all the components fairly well. The structural JCR1 model, which depends on the JDJ model for autocorrelations, predicts the components in the outer region $(y / \delta>0.05)$ better than JDJ, but under-predicts in magnitude the near-wall negative peak in the 1222 component.

Third-order velocity-pressure-gradient has a reduced magnitude in the near-wall region due to straining. The model of DL1 and the JCR2 approach both predict this reduction to some degree; however, the errors in the outer layer become more noticeable and the 111 component is particularly poorly predicted near the wall (figure 20). Models NT and DL2 provide a better prediction of $\Pi_{122}$ in strain than in the unstrained case. This could be caused by a reduced contribution from the 'fast' part, due to lower strain. The KSK2 model predicts almost zero $\Pi_{i j l}$ under applied strain, which is incorrect even near the channel center.

The fourth-order velocity-pressure-gradient magnitudes are decreased by about a factor of two by the effects of straining. The model predictions for the strained case (figure 21) are similar in character to the unstrained case, but appear to be somewhat improved relative to DNS.

Like in the unstrained channel, third-order dissipation is a small contribution to the budget in the strained channel case as well (figure 5). The anisotropic model DL3 predicts a reduction in magnitude of $\varepsilon_{i j l}$; however, 

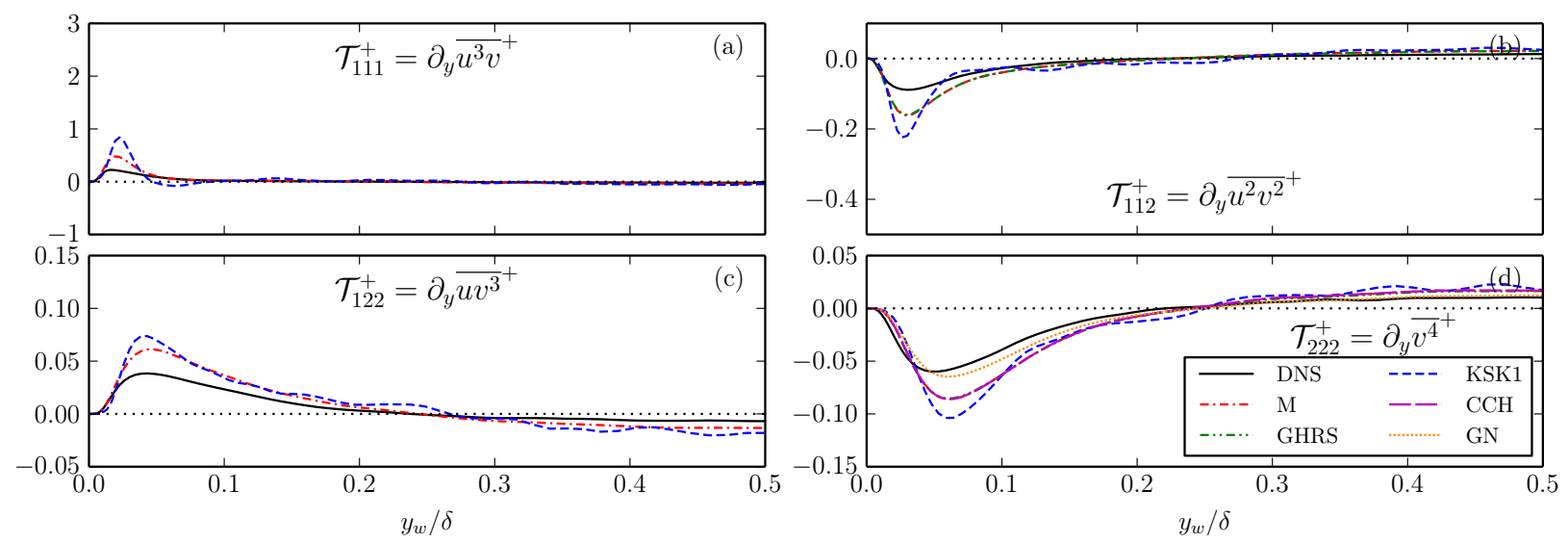

Figure 18. A priori model predictions of third-order turbulence transport term $\left(\mathcal{T}_{i j l}\right)$ in a strained channel at $A_{22} t=0.365$. The terms are non-dimensionalized by $u_{\tau}^{4}(0) / \nu$. The reference statistics from DNS are shown as a solid line.
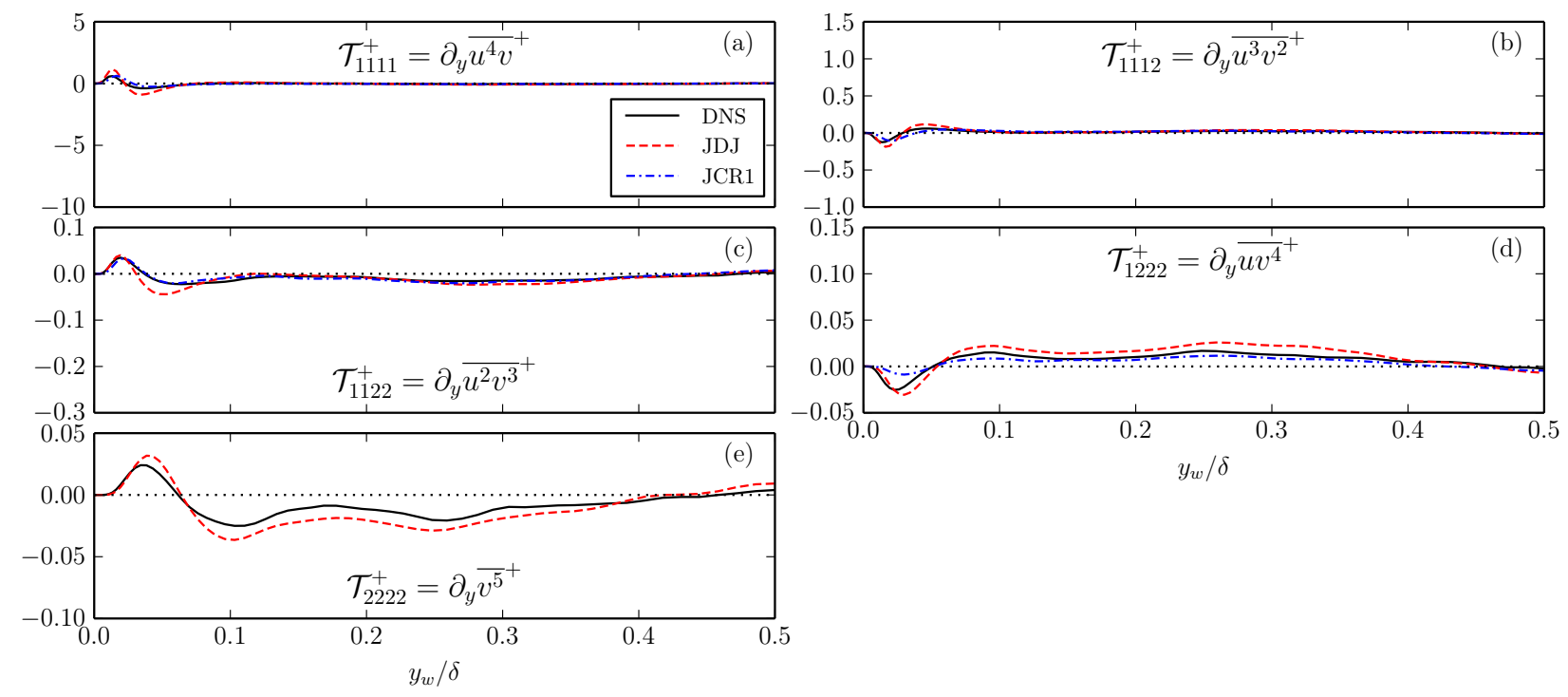

Figure 19. A priori model predictions of fourth-order turbulence transport term $\left(\mathcal{T}_{i j l m}\right)$ in a strained channel at $A_{22} t=0.365$. The terms are non-dimensionalized by $u_{\tau}^{5}(0) / \nu$. The reference statistics from DNS are shown as a solid line. 

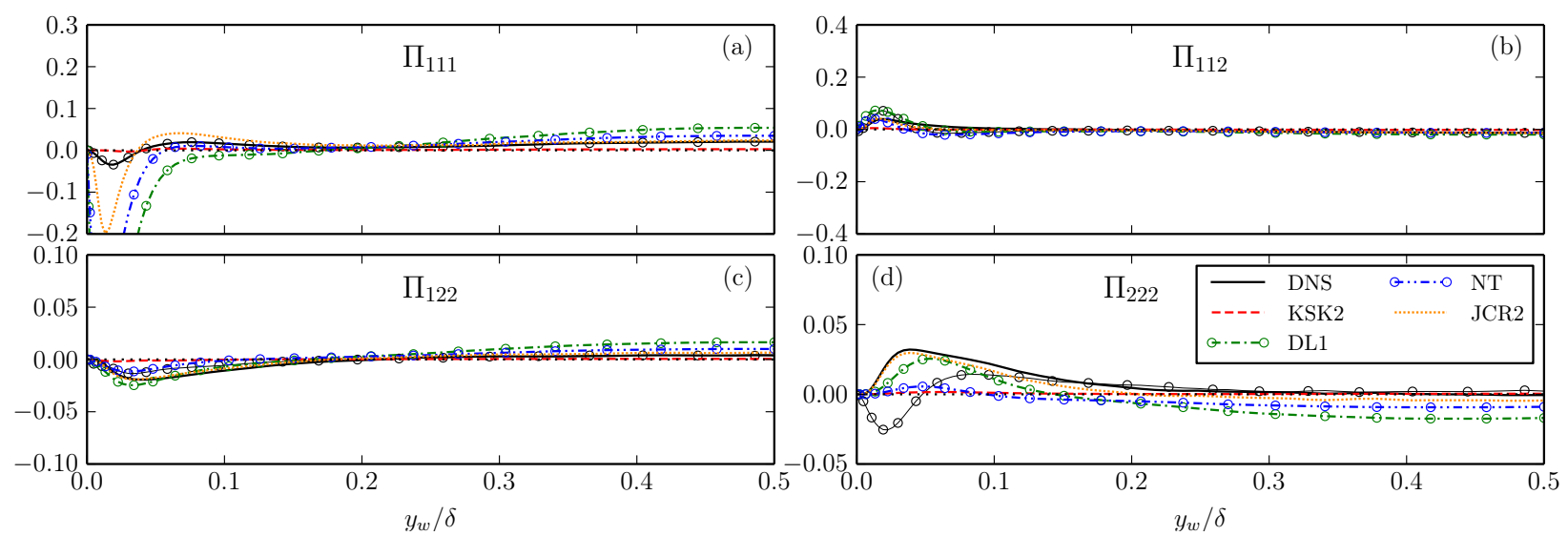

Figure 20. A priori model predictions of third-order velocity-pressure-gradient budget term $\left(\Pi_{i j l}\right)$ in a strained channel at $A_{22} t=0.365$. The terms are non-dimensionalized by $u_{\tau}^{4}(0) / \nu$. The reference $\Pi_{i j l}$ from DNS is shown as a solid line, with pressure-strain term, $\phi_{i j l}$ as $-\odot$.
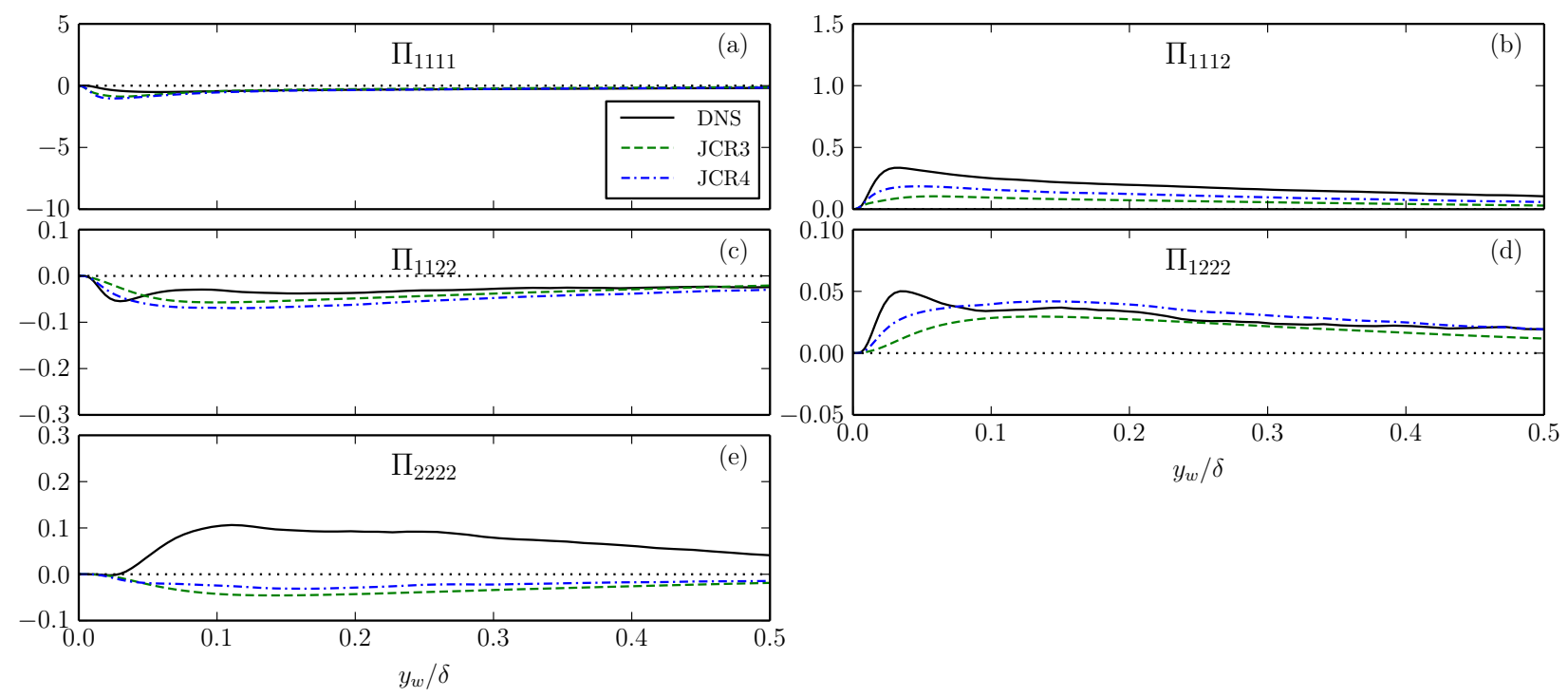

Figure 21. A priori model predictions of fourth-order velocity-pressure-gradient budget term $\left(\Pi_{i j l m}\right)$ in a strained channel at $A_{22} t=0.365$. The terms are non-dimensionalized by $u_{\tau}^{5}(0) / \nu$. The reference statistics from DNS are shown as a solid line. 
it is insufficient to reflect the reference DNS statistics (figure 22). Near wall prediction of $\varepsilon_{222}$ by DL3 has the same magnitude as unstrained data, indicating limited response by the model. The scale-similar KSK3 model responds adequately to the applied strain, and predicts $\varepsilon_{i j l}$ reasonably well.

Fourth-order dissipation budget terms reduce in magnitude significantly due to the effects of applied straining. The JCR5,6 models predict this behavior reasonably well, indicating that $\varepsilon_{i j l m}$ remains scalesimilar under straining conditions. As with the unstrained channel, the KP2 model largely mis-represents the fourth-order dissipation terms.
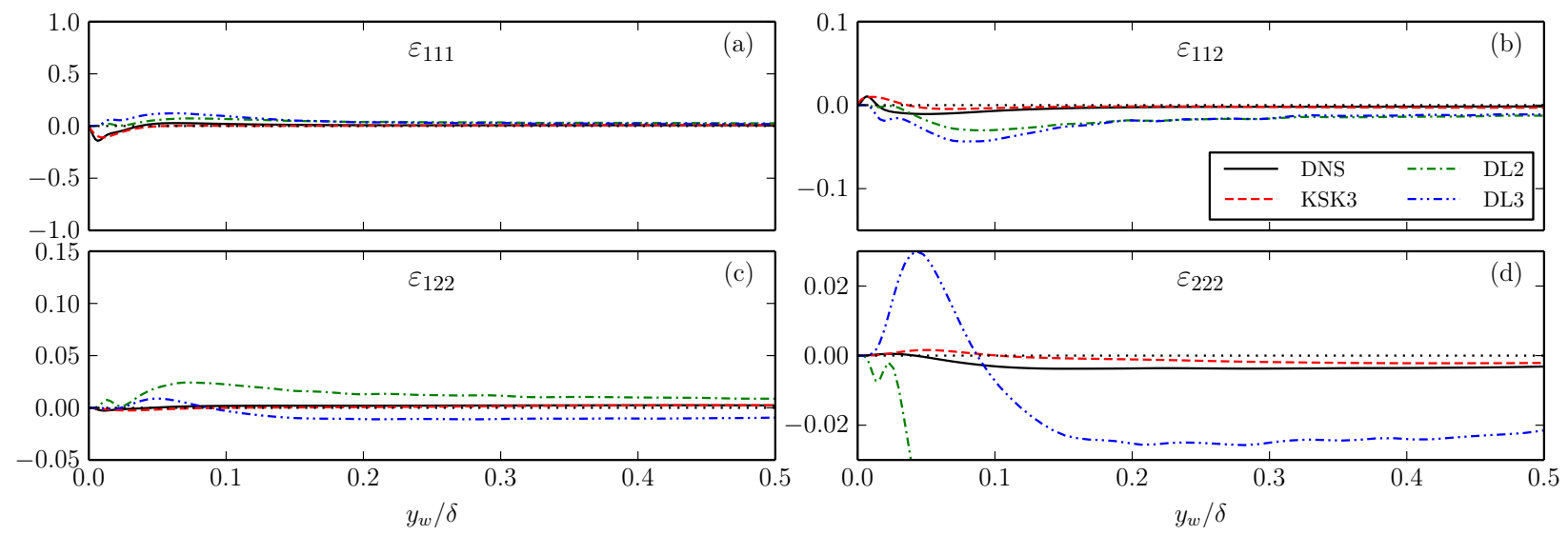

Figure 22. A priori model predictions of third-order dissipation budget term $\left(\varepsilon_{i j l}\right)$ in a strained channel at $A_{22} t=0.365$. The terms are non-dimensionalized by $u_{\tau}^{4}(0) / \nu$. The reference statistics from DNS are shown as a solid line.
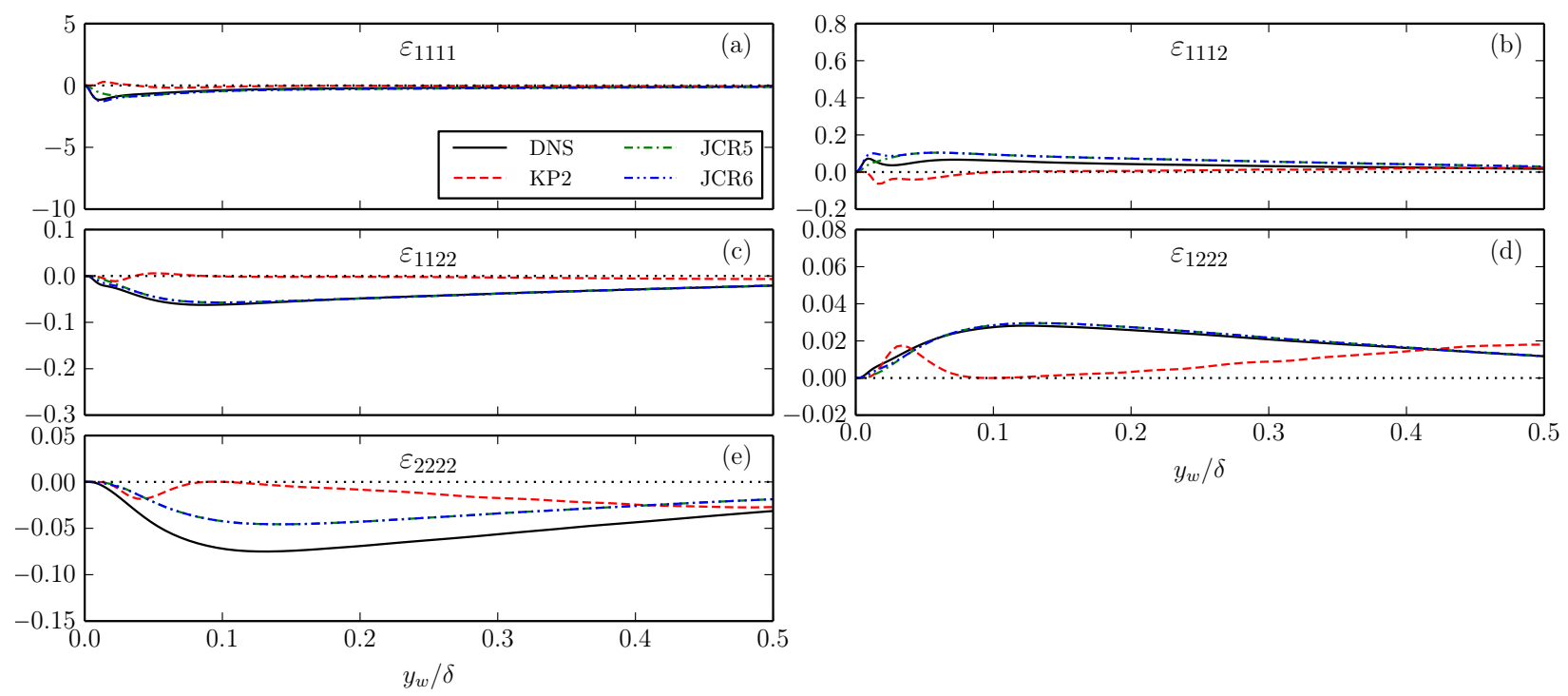

Figure 23. A priori model predictions of fourth-order dissipation budget term $\left(\varepsilon_{i j l m}\right)$ in a strained channel at $A_{22} t=0.365$. The terms are non-dimensionalized by $u_{\tau}^{5}(0) / \nu$. The reference statistics from DNS are shown as a solid line. JCR5 and JCR6 model predictions overlap in all regions except near the wall.

\section{Summary}

A DNS database for third- and fourth-order moment RANS closures has been presented for unstrained and strained (adverse pressure gradient) channel flow. The current DNS, whose geometry has been defined to allow efficient testing of RANS schemes applied to APG flows, agrees well with previously published statistics of second-order moment data, and includes complete budget terms for the higher-order moments. A survey of closure models for higher-order moments was then performed. Both existing models from the literature as 
well as a few newly introduced ideas have been described. Most of the models for $\mathcal{T}$ and $\varepsilon$ relate the budgets to their lower-order moments through a gradient diffusion hypothesis. Velocity-pressure-gradient models are based on analogies to the second-order velocity-pressure-gradient model of a 'slow' return-to-isotropy as well as a 'fast' part.

The extensions of these modeling ideas to higher orders have then been tested via a priori studies of unstrained and strained channel flow, compared to the DNS. For third-order turbulent transport, the model of Millionshtchikov performed fairly well, although it missed some peak values. Modifications to this model fared no better, and in some cases were significantly worse. For fourth-order turbulent transport, both the model of Jovanović et al. as well as a new model introduced here performed about equally well overall, in good agreement with DNS. For the third-order velocity-pressure-gradient, all models fared poorly in general; based on studies using an approximated balance approach, similar difficulties are expected for fourth-order as well. For third-order dissipation, the model of Kawamura et al. was far superior to the other models investigated. For fourth-order dissipation, a new model based on a similar idea (assuming similarity of dissipation to the fourth-order moment) yielded the best agreement with the DNS.

It is recognized that a priori studies are often of limited practical value, and a posteriori tests of an entire scheme is required to form a complete picture. Nonetheless, these current tests can perhaps be used as a guide to modelers to help them choose which model components might be most beneficial to forming a complete scheme. Also, the study demonstrates the particular need for improved model representation of the velocity-pressure-gradient terms. Modeling the second-order velocity-pressure-gradient is already known to be problematic; this difficulty clearly extends to higher-order moments as well.

\section{Appendix A: Near-wall asymptotics}

Very close to a solid wall the spatial rates of change of instantaneous velocity in the streamwise and spanwise directions are small compared with the steep variation normal to the wall. The fluctuating velocity can be thus conveniently expanded in a Taylor series in terms of wall-normal distance $y$ (which, for convenience, replaces the $y_{w}$ used in the main text) as

$$
\begin{aligned}
& u=a_{1} y+a_{2} y^{2}+a_{3} y^{3}+\ldots \\
& v=b_{1} y+b_{2} y^{2}+b_{3} y^{3}+\ldots
\end{aligned}
$$

where coefficients $a_{i}$ and $b_{i}$ are random functions of time and the streamwise $(x)$ and spanwise $(z)$ directions, and have zero mean values. Due to incompressibility, $b_{1}=0$. For a fully developed channel flow, it can be shown that $U \sim y$. This study is similar to the analysis by Lai and $\mathrm{So}^{23}$ for second-moment closure budgets, extended here to third- and fourth-order moments and their budgets. The near-wall limiting behavior of the moments of interest are:

$$
\begin{aligned}
\overline{u^{2}} & =\overline{a_{1}^{2}} y^{2}+\ldots \\
\overline{v^{2}} & =\overline{b_{2}^{2}} y^{4}+\ldots \\
\overline{u v} & =\overline{a_{1} b_{2}} y^{3}+\ldots \\
\overline{u^{2} v} & =\overline{a_{1}^{2} b_{2}} y^{4}+\ldots \\
\overline{u v^{2}} & =\overline{a_{1} b_{2}^{2}} y^{5}+\ldots \\
\overline{v^{3}} & =\overline{b_{2}^{3}} y^{6}+\ldots \\
\overline{u^{3}} & =\overline{a_{1}^{3}} y^{3}+\ldots \\
\overline{u^{3} v} & =\overline{a_{1}^{3} b_{2}} y^{5}+\ldots \\
\overline{u^{2} v^{2}} & =\overline{a_{1}^{2} b_{2}^{2}} y^{6}+\ldots \\
\overline{u v^{3}} & =\overline{a_{1} b_{2}^{3}} y^{7}+\ldots \\
\overline{v^{4}} & =\overline{b_{2}^{4}} y^{8}+\ldots \\
\overline{u^{4}} & =\overline{a_{1}^{4}} y^{4}+\ldots
\end{aligned}
$$

The near-wall behavior of the unclosed budget terms are of importance to developing accurate turbulence models for predicting wall-bounded flows. With the exception of velocity-pressure-gradient $\left(\Pi_{i j l}\right.$ and $\left.\Pi_{i j l m}\right)$ 
terms, the near-wall behavior of every other term in the moment balance equation can be analyzed, as they are only functions of $u_{i}$ and $U_{i}$, which are known. However, the near-wall asymptotic behavior of $\Pi$ can be inferred by summing all the other budget terms and looking at the remainder. From Table A.1, we notice that the velocity-pressure-gradient term has the same asymptotic decay as dissipation and viscous diffusion, except for $\Pi_{111}$ and $\Pi_{1111}$. Hence, similar to the understanding in second-moment near-wall modeling, ${ }^{23}$ to capture the near-wall higher moments, it may be convenient to model the terms $\left(\Pi_{i j l}-\varepsilon_{i j l}\right)$ and $\left(\Pi_{i j l m}-\varepsilon_{i j l m}\right)$.

Table A.1. Wall-limiting behavior of budget terms in the higher-order moment equations

\begin{tabular}{lcccc}
\hline Moment & $\mathcal{P}^{s}, \mathcal{T}, \mathcal{P}^{\tau}$ & $\mathcal{D}$ & $\varepsilon$ & $\Pi$ \\
\hline$\overline{u u}$ & $\mathcal{O}\left(y^{3}\right)$ & $2 \nu \overline{a_{1}^{2}} y^{0}+12 \nu \overline{a_{1} a_{2}} y$ & $-2 \nu \overline{a_{1}^{2}} y^{0}-8 \nu \overline{a_{1} a_{2}} y$ & $\mathcal{O}(y)$ \\
$\overline{v v}$ & $\mathcal{O}\left(y^{5}\right)$ & $12 \nu \overline{b_{2}^{2}} y^{2}+40 \nu \overline{b_{2} b_{3}} y^{3}$ & $-8 \nu \overline{b_{2}^{2}} y^{2}-24 \nu \overline{b_{2} b_{3}} y^{3}$ & $\mathcal{O}\left(y^{2}\right)$ \\
$\overline{u v}$ & $\mathcal{O}\left(y^{4}\right)$ & $6 \nu \overline{a_{1} b_{2}} y+12 \nu \overline{a_{2} b_{2}} y^{2}$ & $-4 \nu \overline{a_{1} b_{2}} y-8 \nu \overline{a_{2} b_{2}} y^{2}$ & $\mathcal{O}(y)$ \\
$\overline{u u u}$ & $\mathcal{O}\left(y^{4}\right)$ & $6 \nu \overline{a_{1}^{3}} y+36 \nu \overline{a_{1}^{2} a_{2}} y^{2}$ & $-6 \nu \overline{a_{1}^{3}} y-6 \nu \overline{a_{1}^{2} a_{2}} y^{2}$ & $\mathcal{O}\left(y^{2}\right)$ \\
$\overline{u u v}$ & $\mathcal{O}\left(y^{5}\right)$ & $12 \nu \overline{a_{1}^{2} b_{2}} y^{2}+20 \nu\left(\overline{a_{1}^{2} b_{3}}+\overline{a_{1} a_{2} b_{2}}\right) y^{3}$ & $-\left(12 \nu \overline{a_{1}^{2} b_{2}}+16 \nu \overline{a_{1} a_{2} b_{2}}\right) y^{2}-16 \nu \overline{a_{1} a_{2} b_{2}} y^{3}$ & $\mathcal{O}\left(y^{2}\right)$ \\
$\overline{u v v}$ & $\mathcal{O}\left(y^{6}\right)$ & $20 \nu \overline{a_{1} b_{2}^{2}} y^{3}+30 \nu\left(\overline{a_{1} b_{2} b_{3}}+\overline{a_{2} b_{2}^{2}}\right) y^{4}$ & $-16 \nu \overline{a_{1} b_{2}^{2}} y^{3}-24 \nu \overline{a_{2} b_{2}^{2}} y^{4}$ & $\mathcal{O}\left(y^{3}\right)$ \\
$\overline{v v v}$ & $\mathcal{O}\left(y^{7}\right)$ & $\left.30 \nu \overline{b_{2}^{3}} y^{4}+42 \nu \overline{\left(b_{2}^{2} b_{3}\right.}+2 \overline{b_{2}^{2} b_{3}}\right) y^{5}$ & $-24 \nu \overline{b_{2}^{3}} y^{4}-72 \nu \overline{b_{2}^{2} b_{3}} y^{5}$ & $\mathcal{O}\left(y^{4}\right)$ \\
$\overline{u u u u}$ & $\mathcal{O}\left(y^{5}\right)$ & $\overline{1} 2 \nu \overline{a_{1}^{4}} y^{2}+80 \nu \overline{a_{1}^{3} a_{2}} y^{3}$ & $-12 \nu \overline{a_{1}^{4}} y^{2}-72 \nu \overline{a_{1}^{3} a_{2}} y^{3}$ & $\mathcal{O}\left(y^{3}\right)$ \\
$\overline{u u u v}$ & $\mathcal{O}\left(y^{6}\right)$ & $20 \nu \overline{a_{1}^{3} b_{2}} y^{3}+30 \nu\left(\overline{a_{1}^{3} b_{3}}+2 \overline{a_{1}^{2} a_{2} b_{2}}\right) y^{4}$ & $-12 \nu \overline{a_{1}^{3} b_{2}} y^{3}-\left(18 \nu \overline{a_{1}^{3} b_{3}}+12 \nu \overline{a_{1}^{2} a_{2} b_{2}}\right) y^{4}$ & $\mathcal{O}\left(y^{3}\right)$ \\
$\overline{u u v v}$ & $\mathcal{O}\left(y^{7}\right)$ & $30 \nu \overline{a_{1}^{2} b_{2}^{2}} y^{4}+42 \nu\left(2 \overline{a_{1}^{2} b_{2} b_{3}}+\overline{a_{1} a_{2} b_{2}^{2}}\right) y^{5}$ & $-26 \nu \overline{a_{1}^{2} b_{2}^{2}} y^{4}-40 \nu \overline{a_{1} a_{2} b_{2}^{2}} y^{5}$ & $\mathcal{O}\left(y^{4}\right)$ \\
$\overline{u v v v}$ & $\mathcal{O}\left(y^{8}\right)$ & $42 \nu \overline{a_{1} b_{2}^{3}} y^{5}+56 \nu\left(\overline{a_{1} b_{2}^{2} b_{3}}+\overline{a_{2} b_{2}^{3}}\right) y^{6}$ & $-36 \nu \overline{a_{1} b_{2}^{3}} y^{5}-80 \nu \overline{a_{1} b_{2}^{3}} y^{6}$ & $\mathcal{O}\left(y^{5}\right)$ \\
$\overline{v v v v}$ & $\mathcal{O}\left(y^{9}\right)$ & $\left.56 \nu \overline{b_{2}^{4}} y^{6}+144 \nu \overline{b_{2}^{3} b_{3}} y^{7}\right)$ & $-48 \nu \overline{b_{2}^{4}} y^{6}-\left(96 \nu \overline{b_{2}^{3} b_{3}}+144 \nu \overline{b_{2}^{4}}\right) y^{7}$ & $\mathcal{O}\left(y^{6}\right)$ \\
\hline
\end{tabular}

\section{Acknowledgments}

The research is supported by NASA Cooperative agreement NNL09AA00A at Langley Research Center awarded to the National Institute of Aerospace. Computational resources were provided by NASA Advanced Supercomputing division. The work of the second and third authors is supported by the Aeronautical Sciences Project of NASA's Fundamental Aeronautics Program. The authors thank S. Woodruff and P. Balakumar of NASA Langley for their helpful comments.

\section{References}

${ }^{1}$ André, J. C., De Moor, G., Lacarrere, P., and Du Vachat, R., "Modeling the 24-Hour Evolution of the Mean and Turbulent Structures of the Planetary Boundary Layer," Journal of the Atmospheric Sciences, Vol. 35, No. 10, 1978, pp. 1861-1883.

${ }^{2}$ Hanjalić, K. and Launder, B., Modelling Turbulence in Engineering and the Environment: Second-Moment Routes to Closure, Cambridge University Press, 2011.

${ }^{3}$ Dekeyser, I. and Launder, B. E., "A Comparison of Triple-Moment Temperature-Velocity Correlations in the Asymmetric Heated Jet with Alternative Closure Models," Turbulent Shear Flows 4, Springer Berlin Heidelberg, 1985, pp. $102-117$.

${ }^{4}$ Kurbatskii, A. F. and Poroseva, S. V., "Modeling Turbulent Diffusion in a Rotating Cylindrical Pipe Flow," Intl. Journal of Heat and Fluid Flow, Vol. 20, 1999, pp. 341-348.

${ }^{5}$ Millionshtchikov, M. D., "On the Theory of Homogeneous Isotropic Turbulence," C. R. Acad. Sci. SSSR, Vol. 32, 1941, pp. 615-619.

${ }^{6}$ Jovanović, J., Durst, F., and Johansson, T. G., "Statistical Analysis of the Dynamic Equations for Higher-Order Moments in Turbulent Wall Bounded Flows," Physics of Fluids A: Fluid Dynamics, Vol. 5, 1993, pp. 2886-2900.

${ }^{7}$ Coleman, G. N., Kim, J., and Spalart, P. R., "Direct Numerical Simulation of a Decelerated Wall-Bounded Turbulent Shear Flow," Journal of Fluid Mechanics, Vol. 495, No. 1, 2003, pp. 1-18.

${ }^{8}$ NASA Langley Turbulence Modeling Resource website, http://turbmodels.larc.nasa.gov, accessed 03/21/2014.

${ }^{9}$ Yorke, C. P. and Coleman, G. N., "Assessment of Common Turbulence Models for an Idealised Adverse Pressure Gradient Flow," European Journal of Mechanics B/Fluids, Vol. 23, 2004, pp. 319-337.

${ }^{10}$ Sciberras, M. A. and Coleman, G. N., "Testing of Reynolds-stress-transport Closures by Comparison with DNS of an Idealized Adverse-pressure-gradient Boundary Layer," European Journal of Mechanics B/Fluids, Vol. 26, 2007, pp. 551-582.

${ }^{11}$ Nagano, Y. and Houra, T., "Higher-order Moments and Spectra of Velocity Fluctuations in Adverse-pressure-gradient Turbulent Boundary Layer," Experiments in Fluids, Vol. 33, 2002, pp. 22-30.

${ }^{12}$ Moser, R. D., Kim, J., and Mansour, N. N., "Direct Numerical Simulation of Turbulent Channel Flow up to Re $\mathrm{e}_{\tau}=590$," Physics of Fluids, Vol. 11, 1999, pp. 943-945. 
${ }^{13}$ Rotta, J., "Statistische Theorie nichthomogener Turbulenz," Z. Phys., Vol. 129, 1951, pp. 547-572.

${ }^{14}$ Nagano, Y. and Tagawa, M., "Turbulence Model for Triple Velocity and Scalar Correlations," Turbulent shear flow 7, Stanford University, August 21-23 1989, pp. 47-62.

${ }^{15}$ Batten, J., "Irreducible Invariants of Fourth-order Tensors," Mathematical Modelling, Vol. 8, 1987, pp. 29-33.

${ }^{16}$ Kawamura, H., Sasaki, J., and Kobayashi, K., "Budget and Modelling of Triple-moment Velocity Correlations in a Turbulent Channel Flow Based on DNS," 10th Symposium on Turbulent Shear Flows, August 14-16, 1995, pp. 13-18.

${ }^{17}$ Cheng, Y., Canuto, V. M., and Howard, A. M., "Nonlocal Convective PBL Model Based on New Third- and Fourth-order Moments," Journal of Atmospheric Science, Vol. 62, July 2005, pp. 2189-2204.

${ }^{18}$ Gryanik, V. M., Hartmann, J., Raasch, S., and Schröter, M., "A Refinement of the Millionshchikov Quasi-normality Hypothesis for Convective Boundary Layer Turbulence," Journal of Atmospheric Sciences, Vol. 62, 2005, pp. 2632-2638.

${ }^{19}$ Grossman, S. A. and Narayan, R., "A Theory of Nonlocal Mixing-length Convection. 2: Generalized Smoothed Particle Hydrodynamics Simulations," Astrophysical Journal Supplement Series, Vol. 89, 1993, pp. 361-394.

${ }^{20}$ Kurbatskii, A. F. and Poroseva, S. V., "A Model for Calculating the Three Components of the Excess for the Turbulent Field of Flow Velocity in a Round Pipe Rotating About its Longitudinal Axis," High Temperature, Vol. 35, No. 3, 1997, pp. $432-440$.

${ }^{21}$ Daly, B. J. and Harlow, F. H., "Transport Equations in Turbulence," Physics of Fluids, Vol. 13, 1970, pp. 2634-2649.

${ }^{22}$ Nagano, Y. and Tagawa, M., "A Structural Turbulence Model for Triple Products of Velocity and Scalar," Journal of Fluid Mechanics, Vol. 215, 1990, pp. 639-657.

${ }^{23}$ Lai, Y. G. and So, R. M. C., "On Near-wall Turbulent Flow Modelling," Journal of Fluid Mechanics, Vol. 221, 1990, pp. 641-673. 